\title{
RECEVEO \\ J111 14997 \\ USTI \\ Batteries for Electric Drive Vehicles: Evaluation of Future \\ Characteristics and Costs through a Delphi Study
}

Anant D. Vyas, Henry K. Ng, Danilo J. Santini, and John L. Anderson

Center for Transportation Research

Argonne National Laboratory

9700 South Cass Avenue, Argonne, Illinois 60439

(630) $252-7578$ (voice)

(630) 252-3443 (fax)

avyas@anl.gov

Paper No. 971628

For Presentation at

the 1997 SAE International Spring Fuels \& Lubricants Meeting

May 5-7, 1997

\begin{abstract}
The submitted manuscript has been authored by a contractor of the U.S. Government under contract No. W-31-109-ENG-3B. under contract No. Wr-31-109-ENG-3B. Accordingly, the U.S. Government retains a nonexclusive, royalty-free license to publish or reproduce the published form of this contribution, or allow others to do so, for U.S. Government purposes.
\end{abstract}

\section{DISCLAIMER}

This report was prepared as an account of work sponsored by an agency of the United States Government. Neither the United States Government nor any agency thereof, nor any of their employees, makes any warranty, express or implied, or assumes any legal liability or responsibility for the accuracy, completeness, or usefulness of any information, apparatus, product, or process disclosed, or represents that its use would not infringe privately owned rights. Reference herein to any specific commercial product, process, or service by trade name, trademark, manufacturer, or otherwise does not necessarily constitute or imply its endorsement, recommendation, or favoring by the United States Government or any agency thereof. The views and opinions of authors expressed herein do not necessarily state or reflect those of the United States Government or any agency thereof.

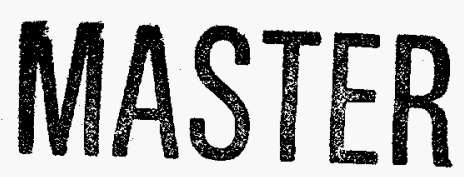




\section{DISCLAIMER}

Portions of this document may be illegible in electronic image products. Images are produced from the best available original document. 


\title{
Batteries for Electric Drive Vehicles: Evaluation of Future Characteristics and Costs through a Delphi Study
}

\author{
Anant D. Vyas, Henry K. Ng, Danilo J. Santini, and John L. Anderson \\ Center for Transportation Research Argonne National Laboratory
}

\section{ABSTRACT}

Uncertainty about future costs and operating attributes of electric drive vehicles (EVs and HEVs) has contributed to considerable debate regarding the market viability of such vehicles. One way to deal with such uncertainty, common to most emerging technologies, is to pool the judgments of experts in the field. Data from a two-stage Delphi study are used to project the future costs and operating characteristics of electric drive vehicles. The experts projected basic vehicle characteristics for EVs and HEVs for the period 20002020. They projected the mean EV range at $179 \mathrm{~km}$ in $2000,270 \mathrm{~km}$ in 2010 , and $358 \mathrm{~km}$ in 2020 . The mean HEV range on battery power was projected as $145 \mathrm{~km}$ in $2000,212 \mathrm{~km}$ in 2010 , and $244 \mathrm{~km}$ in 2020 . Experts' opinions on 10 battery technologies are analyzed and characteristics of initial battery packs for the mean power requirements are presented. A procedure to compute the cost of replacement battery packs is described, and the resulting replacement costs are presented. Projected vehicle purchase prices and fuel and maintenance costs are also presented. The vehicle purchase price and curb weight predictions would be difficult to achieve with the mean battery characteristics. With the battery replacement costs added to the fuel and maintenance costs, the conventional ICE vehicle is projected to have a clear advantage over electric drive vehicles through the projection period.

\section{INTRODUCTION}

As a part of the government's efforts to support research that reduces petroleum consumption, the Office of Transportation Technologies (OTT) within the U.S. Department of Energy (DOE) funds programs leading to increased vehicle fuel economy and use of alternative fuels. A secondary goal is the development of technologies that are less harmful to the environment, as required by legislation and stricter vehicular emission standards. Some of the OTT-funded research provides an impetus to the development of technologies that can improve air quality, particularly in large urban areas, and also alleviate concerns related to global warming associated with burning of fossil fuels. Air quality in the nation's urban areas has been slowly improving, but attainment of standards has proven especially difficult in some urban areas. To some degree, there is concern about diminished air quality in major urban areas in the future due to growth in vehicle use. Federal and state governments therefore continue to develop regulations providing for even stricter vehicle emission standards. The states with the worst problems have contemplated regulations requiring vehicles with zero and ultra-low tailpipe emissions. As a consequence of these developments, OTT has funded research to develop such vehicles, which are mandated by some states.

Electric and hybrid electric vehicles are viewed as solutions to the urban air quality problem of tropospheric (near-ground) ozone. They are also being considered for the purpose of reducing combustion particulates, the damage from which is suspected by some to exceed that from ozone. Because they will use energy stored in batteries or other storage devices, such as ultra-capacitors and flywheels, electric vehicles (EVs) have no tailpipe emissions and are classified as zero emissions vehicles (ZEVs). While EVs have the potential to reduce emissions of pollutants that are precursors to ozone, the technology is unproven, expensive, and can benefit greatly from research and development. Current batteries, EVs' most viable energy storage system, are expensive and bulky, have short cycle life, and also have low energy storage capacity, resulting in limited vehicle range. Advanced battery technologies require extensive research and development efforts, which are being conducted under the auspices of the U.S. Advanced Battery Consortium (USABC). Candidate hybrid electric 
vehicles (HEVs), which are less developed than EVs, are anticipated to use both electrical energy from batteries and power from an onboard system consisting of a gas turbine (GT), an internal combustion engine (ICE), or a fuel cell (FC). An HEV can be a range extender, first using the battery power until it is exhausted and then switching to a power unit for the remainder of the trip. Among HEVs, range extenders usually have the smallest power units. In an alternative configuration, an HEV is powered by a power unit with considerably less power than a conventional vehicle, and peak power is supplied from power stored in the batteries. In this configuration, batteries are charged when the pattern of driving requires less power than what the power unit can provide and through regenerative braking. This is far more beneficial to overall energy use when driving includes many stops and starts. An HEV has the potential to significantly increase the vehicle fuel economy and thereby reduce petroleum consumption and greenhouse gas emissions. However, its two energy storage and power systems make it complex and expensive.

EV and HEV research and development are conducted with public and private funds. Publication of private-sector research and some public-sector research, conducted under agreements that results will remain proprietary, has tended to provide only limited and sketchy details. Battery research has attracted considerable attention because the EV's limited range is viewed as the major barrier to its market viability. However, published information is limited. The battery is also a significant component of EV and HEV costs. Research on electric drive systems that will benefit both the EV and HEV has been under way for some time in both the public and private sectors. Although vehicle manufacturers have not mass-produced either an EV or an HEV, they have recently shown some willingness, due to intense regulatory pressure, to market them in the future. Since comprehensive information on EV and HEV technologies is not readily available, DOE and OTT have sought ways to acquire such information.

In order to obtain a better understanding of the state of both EV and HEV technologies, OTT sponsored a two-stage Delphi study. Argonne National Laboratory (ANL) conducted this study, with assistance from the Society of Automotive Engineers (SAE) Cooperative Research Program (CRP). The goal of the study was to collect information on vehicle attributes and components, evaluate their performance, and assess their marketpenetration potential. The first step was to survey the experts in the field and solicit their opinions. The results of the study will help decision makers to properly orient their research and development efforts.

Information regarding study methodology and questionnaire development has been published earlier [Ng, Anderson, and Santini, 1995; $\mathrm{Ng}$ et al., 1996]. In all, 93 valid second-stage responses were available for analysis. Industry was the largest responding group, providing $47 \%$ of the responses. Within the industry group, original equipment manufacturers provided nearly half the responses (23\% of the total). Private research organizations and potential component suppliers provided $29 \%$ of the responses, and government and academic institutions provided the remainder.

This paper presents an analysis of vehicle attributes and battery technology data from the Delphi study. Ten battery technologies are evaluated, and battery replacement costs for EVs and HEVs are presented. Characteristics of EVs and HEVs that emerged from this analysis are summarized. The vehicle and battery characteristics presented here are taken from the survey; the authors have not attempted to develop any vehicle or battery specifications.

\section{ELECTRIC DRIVE VEHICLE CHARACTERISTICS}

The survey was designed to collect information for the years 2000,2010 , and 2020 . The questionnaire contained sections on vehicles, components, and system impacts. Each section contained questions seeking opinions on critical characteristics of EVs and HEVs. Some questions within the vehicles section sought experts' opinions on such vehicle attributes as range, acceleration, highest acceptable uphill grade, seating capacity, cargo capacity, curb weight, power, battery recharging time, and maintenance interval. For the HEV, an additional question was asked concerning the engine range. We computed the implied battery-only range by subtracting engine range from the total HEV range. Experts also provided opinions on EV and HEV price, fuel and maintenance cost, and fuel economy. Within the components section of the questionnaire, they responded to questions about 10 battery technologies. We analyzed the responses to all these questions to arrive at two separate estimates of EV and HEV characteristics, primary (i.e., resulting from respondents' vehicle opinions) and secondary (i.e., resulting from respondents' battery opinions).

For most of the analysis presented here, we have used mean values of the responses. We have listed several other values, such as number of valid responses, median, and mode, whenever we summarize the responses in a table. We also present optimistic and pessimistic values for the basic vehicle characteristics. These values represent means of the responses either below and at the median point or above the median point (i.e., the responses are split into two groups at the median point). A group's optimistic or pessimistic identity is dependent on the attribute. For example, because longer range and lower vehicle curb weight are desirable, the optimistic group for range will be above the median and the optimistic group for curb weight will be at and below the median. A good measure of the level of agreement among the respondents is the interquartile range. A narrower interquartile range represents a 
higher degree of agreement. We also present these statistics for the data summarized in tables.

BASIC VEHICLE CHARACTERISTICS - The basic vehicle characteristics for EVS and HEVs are power, curb weight, seating capacity, cargo capacity, and range. These characteristics are summarized in Table 1. Since events subsequent to initiation of the study appear to have pushed back the likely introduction date for EVs past 2000, the table contains values for the year 2005, which we derived through linear interpolation. As can be seen from the number of observations, most respondents answered the basic vehicle characteristics questions. They project consistent improvements in both the EV and the HEV. Other characteristics on which opinions were requested but are not summarized in Table 1 include acceleration, recharging time, and maintenance interval [ $\mathrm{Ng}$ et al., 1996]. Such attributes as acceleration, top speed, and the ability to negotiate a reasonable uphill grade are dependent on vehicle design and power. Responses to recharging time and maintenance interval queries are summarized in a separate publication [ANL, 1996].

The mean EV range is $179 \mathrm{~km}$ in the year 2000 , increasing to $270 \mathrm{~km}$ by 2010 (a $51 \%$ increase) and to $358 \mathrm{~km}$ by 2020 (a 100\% increase). The mean EV power is $66.7 \mathrm{~kW}$ in the year 2000, $86.1 \mathrm{~kW}$ in 2010 (a $29 \%$ increase), and $99.2 \mathrm{~kW}$ in 2020 (a $49 \%$ increase over 2000). The respondents appear to have recognized that most batteries would have to improve less to provide the needed power, compared to the extent of improvements needed in specific energy to obtain the desired range. Experts appear to have provided the peak power rating for the motor, rather than the continuous power rating, and did not seem to foresee substantial improvements in EV power between now and 2000. At present, a typical $A C$ induction motor is rated at approximately $66 \mathrm{~kW}$ peak power, and a DC motor, around 52 kW [Cuenca and Gaines, 1996].

An $E V$ is projected to have a mean curb weight of $1,538 \mathrm{~kg}$ in $2000,1,351 \mathrm{~kg}$ in 2010 (a $12 \%$ reduction), and $1,222 \mathrm{~kg}$ in 2020 (a $21 \%$ reduction). A mid-size conventional car had a curb weight of $1,368 \mathrm{~kg}$ in 1993. While its curb weight in 2010 is comparable with that of today's conventional mid-size car, the future EV is not likely to have the same seating capacity. A present midsize conventional car's seating capacity is 5 , but the mean seating capacity of an EV is projected at 3 or 4 until 2010. The projected improvements in power, range, and curb weight pose substantial challenges to $E V$ manufacturers. Lightweight materials, such as aluminum and carbon polymer, have the potential to reduce the curb weight, but vehicles that use such materials will cost more. An aluminum-intensive mid-size conventional car would weigh $31 \%$ less when power per unit mass is held constant; however, such a car would cost $\$ 1,200$ (1990 dollars) more [Stodolsky, Vyas, and Cuenca, 1995].
Table 1 also shows the projected attributes of an HEV. Its mean power is projected at $79.6 \mathrm{~kW}$ in 2000 , $99.1 \mathrm{~kW}$ in 2010 (a $24 \%$ increase), and $108.6 \mathrm{~kW}$ in 2020 (a $36 \%$ increase). Three HEV range values are summarized in the table: (1) total, (2) engine, and (3) battery; respondents provided "total" and "engine" ranges, and we computed the "battery" range for each respondent. A large majority (94\%) of the respondents expected the future HEV to be a "range extender." A small battery range indicates that the respondent did not expect the HEV to be a "range extender" because it will not be charged through the electric grid. Only 7 respondents in the year 2000 and 5 respondents each in 2010 and 2020 provided very small battery ranges.

The mean total range of an HEV is projected to be $353 \mathrm{~km}$ in 2000, $469 \mathrm{~km}$ in 2010 (a 33\% increase), and $527 \mathrm{~km}$ in 2020 (a $49 \%$ increase). The total range is less than the range of a conventional vehicle (500-550 $\mathrm{km}$ ) until 2020, which indicates that the respondents did not see HEVs being used for vacations and long trips until after 2010. The mean engine range is $215 \mathrm{~km}$ in $2000,257 \mathrm{~km}$ in 2010 (a $20 \%$ increase), and $281 \mathrm{~km}$ in 2020 (a 31\% increase). The engine range does not increase as much as the total range. The respondents saw the battery supplying more of the HEV range, increasing from $39 \%$ of the total range in 2000 to $47 \%$ in 2020.

The mean curb weight of an HEV is $1,556 \mathrm{~kg}$ in $2000,1,382 \mathrm{~kg}$ in 2010 (a $11 \%$ reduction), and $1,265 \mathrm{~kg}$ in 2020 (a $19 \%$ reduction). The rate of weight reduction is slightly lower than the rate for an EV (see above). The respondents saw an HEV as being slightly heavier than an EV and having one additional seat through 2010.

VEHICLE COSTS - Respondents were asked to provide EV and HEV purchase prices and fuel and maintenance costs relative to the 1993 conventional vehicle. Vehicle purchase prices are summarized in Table 2, and fuel and maintenance costs are summarized in Table 3 . The respondents projected the conventional vehicle to cost $26 \%$ more by $2000,60 \%$ more by 2010 , and $102 \%$ more by 2020 . They expected both EVs and HEVs to cost more than the conventional vehicle through the year 2020. An EV is projected to cost $129 \%$ more than the 1993 conventional vehicle in the year $2000,131 \%$ more in 2010 , and $138 \%$ more in 2020. The least expensive HEV, an ICE-powered version, will cost $147 \%$ more than the 1993 conventional vehicle in $2000,144 \%$ more in 2010 , and $154 \%$ more in 2020. A fuel-cell HEV is projected to be the most expensive of the four electric drive vehicles, costing $415 \%$ more than the 1993 conventional vehicle in 2000 and $298 \%$ more in 2020 . The interquartile ranges are wider for the gas-turbine-powered and fuel-cell-powered HEVs. 
TABLE 1 EV and HEV Basic Vehicle Characteristics

\begin{tabular}{|c|c|c|c|c|c|c|c|c|c|}
\hline Attribute & Year & Obs & Mean & Mode & Median & Qrtl-1 & Qrti-3 & Optimist & Pessimist \\
\hline \multicolumn{10}{|l|}{ Electric Vehicle } \\
\hline \multirow[t]{4}{*}{ Range (km) } & 2000 & 92 & 179 & 150 & 150 & 150 & 250 & 220 & 138 \\
\hline & 2005 & 92 & 225 & 200 & 200 & 194 & 300 & 281 & 169 \\
\hline & 2010 & 92 & 270 & 250 & 250 & 238 & 350 & 341 & 199 \\
\hline & 2020 & 92 & 358 & 250 & 350 & 250 & 450 & 465 & 250 \\
\hline \multirow[t]{4}{*}{ Seats } & 2000 & 91 & 3 & 4 & 4 & 2 & 4 & 4 & 3 \\
\hline & 2005 & 91 & 4 & 4 & 4 & 3 & 4 & 4 & 3 \\
\hline & 2010 & 91 & 4 & 4 & 4 & 4 & 4 & 5 & 4 \\
\hline & 2020 & 90 & 5 & 5 & 5 & 4 & 5 & 5 & 4 \\
\hline \multirow[t]{4}{*}{ Curb weight (kg) } & 2000 & 88 & 1,538 & 1,800 & 1,400 & 1,400 & 1,800 & 1,305 & 1,771 \\
\hline & 2005 & 88 & 1,444 & 1,600 & 1,400 & 1,300 & 1,600 & 1,240 & 1,649 \\
\hline & 2010 & 88 & 1,351 & 1,400 & 1,400 & 1,200 & 1,400 & 1,175 & 1,527 \\
\hline & 2020 & 88 & 1,222 & 1,200 & 1,200 & 1,200 & 1,400 & 1,075 & 1,370 \\
\hline \multirow[t]{4}{*}{ Power (kW) } & 2000 & 91 & 66.7 & 70.0 & 70.0 & 50.0 & 70.0 & 79 & 54 \\
\hline & 2005 & 91 & 76.4 & 80.0 & 80.0 & 60.0 & 80.0 & 90 & 63 \\
\hline & 2010 & 91 & 86.1 & 90.0 & 90.0 & 70.0 & 90.0 & 100 & 72 \\
\hline & 2020 & 90 & 99.2 & 110.0 & 99.8 & 90.0 & 110.0 & 114 & 84 \\
\hline \multicolumn{10}{|c|}{ Hybrid Electric Vehicle } \\
\hline \multirow[t]{4}{*}{ Total range $(\mathbf{k m})$} & 2000 & 91 & 353 & 350 & 350 & 250 & 450 & 420 & 286 \\
\hline & 2005 & 91 & 411 & 400 & 400 & 350 & 450 & 480 & 342 \\
\hline & 2010 & 91 & 469 & 450 & 450 & 450 & 450 & 539 & 398 \\
\hline & 2020 & 91 & 527 & 650 & 450 & 450 & 650 & 628 & 423 \\
\hline \multirow[t]{4}{*}{ Engine range (km) } & 2000 & 88 & 215 & 300 & 200 & 150 & 300 & 286 & 144 \\
\hline & 2005 & 88 & 236 & 300 & 204 & 175 & 300 & 309 & 164 \\
\hline & 2010 & 88 & 257 & 300 & 209 & 200 & 300 & 331 & 183 \\
\hline & 2020 & 88 & 281 & 300 & 300 & 200 & 300 & 345 & 218 \\
\hline \multirow[t]{4}{*}{ Battery range $(\mathrm{km})$} & 2000 & 88 & 145 & 150 & 150 & 100 & 200 & 199 & 90 \\
\hline & 2005 & 88 & 178 & 200 & 178 & 125 & 225 & 246 & 110 \\
\hline & 2010 & 88 & 212 & 250 & 205 & 150 & 250 & 293 & 130 \\
\hline & 2020 & 88 & 244 & 350 & 250 & 150 & 350 & 357 & 152 \\
\hline \multirow[t]{4}{*}{ Seats } & 2000 & 89 & 4 & 4 & 4 & 4 & 4 & 4 & 3 \\
\hline & 2005 & 90 & 4 & 4 & 4 & 4 & 5 & 5 & 4 \\
\hline & 2010 & 88 & 5 & 4 & 4 & 4 & 5 & 5 & 4 \\
\hline & 2020 & 89 & 5 & 5 & 5 & 4 & 6 & 6 & 5 \\
\hline \multirow[t]{4}{*}{ Curb weight (kg) } & 2000 & 85 & 1,556 & 1,800 & 1,400 & 1,400 & 1,800 & 1,338 & 1,769 \\
\hline & 2005 & 84 & 1,469 & 1,600 & 1,400 & 1,300 & 1,600 & 1,277 & 1,659 \\
\hline & 2010 & 84 & 1,382 & 1,400 & 1,400 & 1,200 & 1,400 & 1,215 & 1,548 \\
\hline & 2020 & 85 & 1,265 & 1,200 & 1,200 & 1,200 & 1,400 & 1,112 & 1,415 \\
\hline \multirow[t]{4}{*}{ Power (kW) } & 2000 & 87 & 79.6 & 70.0 & 70.0 & 70.0 & 90.0 & 94 & 65 \\
\hline & 2005 & 86 & 89.4 & 80.0 & 80.0 & 80.0 & 100.0 & 104 & 75 \\
\hline & 2010 & 86 & 99.1 & 90.0 & 90.0 & 90.0 & 110.0 & 113 & 85 \\
\hline & 2020 & 87 & 108.6 & 110.0 & 110.0 & 90.0 & 110.0 & 124 & 94 \\
\hline
\end{tabular}

a The statistics for EV and HEV range have been published earlier (see $\mathrm{Ng}$ et al., 1996). 
TABLE 2 Vehicle Purchase Price (in nominal dollars) for Conventional and Electric Drive Vehicles

\begin{tabular}{lccccccccr} 
Vehicle Technology & Year & Obs & Mean & Mode & \multicolumn{3}{c}{ Median } & Quartile-1 Quartile-3 Optimist Pessimist \\
\hline Conventional ICE & 2000 & 87 & 18,862 & 18,000 & 18,000 & 16,500 & 19,650 & 16,929 & 20,751 \\
& 2005 & 86 & 21,457 & 19,500 & 20,250 & 18,750 & 23,325 & 18,454 & 24,434 \\
& 2010 & 86 & 24,052 & 21,000 & 22,500 & 21,000 & 27,000 & 19,978 & 28,727 \\
& 2020 & 87 & 30,336 & 30,000 & 30,000 & 23,250 & 34,500 & 22,919 & 37,585 \\
Electric vehicle & 2000 & 87 & 34,300 & 30,000 & 30,000 & 30,000 & 40,500 & 26,724 & 41,703 \\
& 2005 & 86 & 34,447 & 30,000 & 31,500 & 30,000 & 39,000 & 27,246 & 41,562 \\
& 2010 & 86 & 34,594 & 30,000 & 33,000 & 30,000 & 37,500 & 27,767 & 41,421 \\
& 2020 & 87 & 35,703 & 30,000 & 33,000 & 30,000 & 37,500 & 27,181 & 44,032 \\
ICE-powered hybrid & 2000 & 85 & 37,082 & 33,000 & 35,250 & 30,000 & 43,500 & 28,025 & 45,928 \\
& 2005 & 84 & 36,817 & 35,250 & 35,625 & 30,000 & 41,663 & 28,070 & 45,459 \\
& 2010 & 84 & 36,552 & 37,500 & 36,000 & 30,000 & 39,825 & 28,114 & 44,989 \\
& 2020 & 85 & 38,051 & 37,500 & 37,500 & 30,000 & 42,000 & 28,339 & 47,536 \\
Gas-turbine-powered hybrid & 2000 & 76 & 49,089 & 45,000 & 45,000 & 37,500 & 60,000 & 34,903 & 63,276 \\
& 2005 & 76 & 47,372 & 45,000 & 45,000 & 37,181 & 53,344 & 34,385 & 60,360 \\
& 2010 & 84 & 45,655 & 45,000 & 45,000 & 36,863 & 46,688 & 33,808 & 57,443 \\
& 2020 & 85 & 46,016 & 45,000 & 45,000 & 36,000 & 48,750 & 33,207 & 58,528 \\
Fuel-cell-powered hybrid & 2000 & 70 & 77,231 & 60,000 & 67,500 & 46,125 & 86,250 & 47,023 & 107,439 \\
& 2005 & 70 & 71,288 & 75,000 & 63,750 & 45,563 & 88,125 & 45,344 & 96,967 \\
& 2010 & 81 & 65,344 & 90,000 & 60,000 & 45,000 & 90,000 & 43,665 & 86,495 \\
& 2020 & 83 & 59,770 & 60,000 & 57,000 & 37,500 & 75,000 & 37,968 & 81,054 \\
\hline
\end{tabular}

TABLE 3 Fuel and Maintenance Costs (nominal cents per $\mathbf{k m}$ ) for Conventional and Electric Drive Vehicles ${ }^{\text {a }}$

\begin{tabular}{|c|c|c|c|c|c|c|c|c|c|}
\hline Vehicle Technology & Year & Obs & Mean & Mode & Median & Quartile-1 & Quartile-3 & Optimist & Pessimist \\
\hline \multirow[t]{4}{*}{ Conventional ICE } & 2000 & 85 & 6.9 & 6.4 & 6.9 & 6.4 & 7.5 & 6.3 & 7.5 \\
\hline & 2005 & 84 & 7.7 & 7.2 & 7.5 & 6.9 & 8.4 & 6.8 & 8.7 \\
\hline & 2010 & 84 & 8.5 & 8.1 & 8.1 & 7.5 & 9.2 & 7.2 & 9.8 \\
\hline & 2020 & 83 & 10.4 & 8.7 & 9.2 & 8.4 & 11.6 & 7.9 & 12.9 \\
\hline \multirow[t]{4}{*}{ Electric vehicle } & 2000 & 84 & 8.2 & 7.5 & 7.5 & 6.4 & 8.7 & 6.1 & 10.3 \\
\hline & 2005 & 83 & 8.2 & 7.2 & 7.5 & 6.6 & 8.7 & 6.2 & 10.3 \\
\hline & 2010 & 83 & 8.3 & 6.9 & 7.5 & 6.9 & 8.7 & 6.2 & 10.4 \\
\hline & 2020 & 83 & 8.9 & 8.7 & 7.5 & 6.2 & 10.4 & 5.8 & 11.9 \\
\hline \multirow[t]{4}{*}{ ICE-powered hybrid } & 2000 & 79 & 9.0 & 8.7 & 8.7 & 7.5 & 9.8 & 7.3 & 10.7 \\
\hline & 2005 & 79 & 9.2 & 8.4 & 8.7 & 7.8 & 10.1 & 7.4 & 11.1 \\
\hline & 2010 & 79 & 9.4 & 8.1 & 8.7 & 8.1 & 10.4 & 7.5 & 11.4 \\
\hline & 2020 & 79 & 10.9 & 11.0 & 10.4 & 8.4 & 11.6 & 8.1 & 13.7 \\
\hline \multirow[t]{4}{*}{ Gas-turbine-powered hybrid } & 2000 & 79 & 11.6 & 8.7 & 10.4 & 8.7 & 11.6 & 8.5 & 14.6 \\
\hline & 2005 & 79 & 11.4 & 9.5 & 10.4 & 8.7 & 11.6 & 8.6 & 14.3 \\
\hline & 2010 & 80 & 11.3 & 10.4 & 10.4 & 8.7 & 11.6 & 8.6 & 14.1 \\
\hline & 2020 & 80 & 12.0 & 10.4 & 10.4 & 8.7 & 12.7 & 8.9 & 15.3 \\
\hline \multirow[t]{4}{*}{ Fuel-cell-powered hybrid } & 2000 & 73 & 15.4 & 11.6 & 12.1 & 11.6 & 17.3 & 9.8 & 21.0 \\
\hline & 2005 & 73 & 14.3 & 11.6 & 11.8 & 10.7 & 15.9 & 9.7 & 18.8 \\
\hline & 2010 & 76 & 13.1 & 11.6 & 11.6 & 9.8 & 14.4 & 9.7 & 16.6 \\
\hline & 2020 & 77 & 13.6 & 11.6 & 11.6 & 9.8 & 14.4 & 9.1 & 18.0 \\
\hline
\end{tabular}

Excludes battery replacement costs for electric drive vehicles. 
TABLE 4 Specific Energy (in Wh/kg) for 10 Battery Technologies

\begin{tabular}{|c|c|c|c|c|c|c|c|}
\hline \multirow{5}{*}{$\frac{\text { Battery Te }}{\text { Lead acid }}$} & & $\mathrm{COS}$ & ean & & & & \\
\hline & 2000 & 55 & 40 & 40 & 40 & 35 & $\overline{40}$ \\
\hline & 2005 & 55 & 42 & 40 & 42 & 38 & 45 \\
\hline & 2010 & 55 & 44 & 40 & 44 & 40 & 50 \\
\hline & 2020 & 54 & 48 & 45 & 45 & 41 & 51 \\
\hline \multirow[t]{4}{*}{ Lithium iron disulfide } & 2000 & 24 & 97 & 100 & 96 & 80 & 100 \\
\hline & 2005 & 22 & 106 & 105 & 104 & 91 & 109 \\
\hline & 2010 & 23 & 116 & 110 & 110 & 100 & 130 \\
\hline & 2020 & 32 & 138 & 150 & 135 & 119 & 150 \\
\hline \multirow[t]{4}{*}{ Lithium polymer } & 2000 & 39 & 110 & 100 & 100 & 100 & 123 \\
\hline & 2005 & 39 & 125 & 125 & 125 & 118 & 135 \\
\hline & 2010 & 41 & 144 & 150 & 150 & 130 & 150 \\
\hline & 2020 & 41 & 172 & 200 & 170 & 150 & 200 \\
\hline \multirow[t]{4}{*}{ Nickel cadmium } & 2000 & 45 & 57 & 55 & 57 & 55 & 60 \\
\hline & 2005 & 44 & 58 & 55 & 58 & 55 & 63 \\
\hline & 2010 & 44 & 60 & 55 & 60 & 55 & 65 \\
\hline & 2020 & 43 & 62 & 55 & 62 & 55 & 66 \\
\hline \multirow[t]{4}{*}{ Nickel iron } & 2000 & 23 & 51 & 50 & 50 & 50 & 55 \\
\hline & 2005 & 22 & 53 & 53 & 53 & 52 & 56 \\
\hline & 2010 & 22 & 55 & 55 & 55 & 53 & 58 \\
\hline & 2020 & 22 & 58 & 60 & 58 & 55 & 60 \\
\hline \multirow[t]{4}{*}{ Nickel metal hydride } & 2000 & 43 & 73 & 75 & 75 & 70 & 75 \\
\hline & 2005 & 43 & 78 & 78 & 78 & 74 & 81 \\
\hline & 2010 & 43 & 83 & 80 & 80 & 80 & 85 \\
\hline & 2020 & 42 & 89 & 85 & 85 & 85 & 95 \\
\hline \multirow[t]{4}{*}{ Nickel zinc } & 2000 & 20 & 61 & 60 & 60 & 55 & 63 \\
\hline & 2005 & 20 & 64 & 63 & 64 & 59 & 67 \\
\hline & 2010 & 20 & 68 & 70 & 70 & 64 & 70 \\
\hline & 2020 & 20 & 74 & 80 & 76 & 67 & 80 \\
\hline \multirow[t]{4}{*}{ Sodium sulfur } & 2000 & 41 & 95 & 80 & 90 & 80 & 110 \\
\hline & 2005 & 40 & 99 & 110 & 95 & 85 & 110 \\
\hline & 2010 & 40 & 102 & 110 & 100 & 90 & 110 \\
\hline & 2020 & 39 & 107 & 110 & 110 & 95 & 110 \\
\hline \multirow[t]{4}{*}{ Zinc air } & 2000 & 29 & 116 & 120 & 120 & 100 & 120 \\
\hline & 2005 & 28 & 127 & 125 & 125 & 110 & 135 \\
\hline & 2010 & 28 & 137 & 150 & 130 & 120 & 150 \\
\hline & 2020 & 27 & 146 & 150 & 150 & 130 & 160 \\
\hline \multirow[t]{4}{*}{ Zinc bromide } & 2000 & 21 & 69 & 70 & 70 & 65 & 70 \\
\hline & 2005 & 21 & 72 & 73 & 71 & 67 & 74 \\
\hline & 2010 & 21 & 75 & 70 & 72 & 70 & 75 \\
\hline & 2020 & 21 & 79 & 80 & 77 & 72 & 80 \\
\hline
\end{tabular}


TABLE 5 Specific Power (in W/kg) for 10 Battery Technologies

Battery Technology Year Obs Mean Mode Median Quartile-1 Quartile-3

\begin{tabular}{|c|c|c|c|c|c|c|c|}
\hline \multirow[t]{4}{*}{ Lead acid } & 2000 & 54 & 155 & 200 & 130 & 100 & 200 \\
\hline & 2005 & 53 & 173 & 200 & 145 & 110 & 200 \\
\hline & 2010 & 53 & 190 & 200 & 160 & 120 & 200 \\
\hline & 2020 & 54 & 214 & 200 & 190 & 120 & 200 \\
\hline \multirow[t]{4}{*}{ Lithium iron disulfide } & 2000 & 22 & 167 & 150 & 155 & 150 & 195 \\
\hline & 2005 & 22 & 188 & 225 & 178 & 159 & 215 \\
\hline & 2010 & 23 & 209 & 300 & 200 & 168 & 235 \\
\hline & 2020 & 32 & 269 & 350 & 235 & 200 & 350 \\
\hline \multirow[t]{4}{*}{ Lithium polymer } & 2000 & 38 & 136 & 150 & 140 & 120 & 150 \\
\hline & 2005 & 38 & 152 & 155 & 150 & 135 & 167 \\
\hline & 2010 & 39 & 167 & 160 & 160 & 150 & 184 \\
\hline & 2020 & 39 & 193 & 180 & 180 & 180 & 209 \\
\hline \multirow[t]{4}{*}{ Nickel cadmium } & 2000 & 43 & 189 & 175 & 180 & 175 & 200 \\
\hline & 2005 & 43 & 194 & 175 & 185 & 175 & 205 \\
\hline & 2010 & 43 & 199 & 175 & 190 & 175 & 210 \\
\hline & 2020 & 43 & 209 & 175 & 200 & 182 & 222 \\
\hline \multirow[t]{4}{*}{ Nickel iron } & 2000 & 22 & 125 & 130 & 130 & 120 & 131 \\
\hline & 2005 & 22 & 133 & 133 & 133 & 125 & 136 \\
\hline & 2010 & 22 & 140 & 135 & 135 & 130 & 140 \\
\hline & 2020 & 22 & 152 & 150 & 150 & 140 & 150 \\
\hline \multirow[t]{4}{*}{ Nickel metal hydride } & 2000 & 41 & 165 & 150 & 155 & 150 & 175 \\
\hline & 2005 & 41 & 174 & 150 & 168 & 150 & 188 \\
\hline & 2010 & 41 & 184 & 150 & 180 & 150 & 200 \\
\hline & 2020 & 41 & 203 & 200 & 200 & 175 & 220 \\
\hline \multirow[t]{4}{*}{ Nickel zinc } & 2000 & 20 & 171 & 150 & 161 & 150 & 175 \\
\hline & 2005 & 20 & 181 & 165 & 171 & 163 & 188 \\
\hline & 2010 & 20 & 192 & 180 & 180 & 175 & 200 \\
\hline & 2020 & 20 & 214 & 200 & 200 & 198 & 231 \\
\hline \multirow[t]{4}{*}{ Sodium sulfur } & 2000 & 40 & 144 & 150 & 142 & 132 & 150 \\
\hline & 2005 & 40 & 149 & 150 & 146 & 140 & 155 \\
\hline & 2010 & 40 & 153 & 150 & 150 & 148 & 160 \\
\hline & 2020 & 40 & 160 & 150 & 150 & 150 & 170 \\
\hline \multirow[t]{4}{*}{ Zinc air } & 2000 & 27 & 91 & 100 & 100 & 83 & 100 \\
\hline & 2005 & 27 & 100 & 100 & 105 & 91 & 110 \\
\hline & 2010 & 27 & 108 & 100 & 110 & 100 & 120 \\
\hline & 2020 & 26 & 122 & 120 & 120 & 113 & 130 \\
\hline \multirow[t]{4}{*}{ Zinc bromide } & 2000 & 21 & 94 & 90 & 90 & 90 & 100 \\
\hline & 2005 & 20 & 102 & 90 & 100 & 92 & 112 \\
\hline & 2010 & 20 & 110 & 90 & 110 & 94 & 124 \\
\hline & 2020 & 21 & 124 & 140 & 130 & 110 & 140 \\
\hline
\end{tabular}


TABLE 6 Shelf Life (in years) for 10 Battery Technologies

\begin{tabular}{|c|c|c|c|c|c|c|c|}
\hline Battery Technology & Year & Obs & Mean & Mode & Median & Quartile-1 & Quartile-3 \\
\hline \multirow[t]{4}{*}{ Lead acid } & 2000 & 50 & 3.4 & 3.0 & 3.0 & 3.0 & 4.0 \\
\hline & 2005 & 49 & 3.9 & 3.5 & 3.5 & 3.0 & 4.3 \\
\hline & 2010 & 49 & 4.4 & 4.0 & 4.0 & 3.0 & 4.6 \\
\hline & 2020 & 49 & 4.4 & 5.0 & 4.5 & 3.0 & 5.0 \\
\hline \multirow[t]{4}{*}{ Lithium iron disulfide } & 2000 & 21 & 3.5 & 4.0 & 4.0 & 3.0 & 4.0 \\
\hline & 2005 & 21 & 4.0 & 4.5 & 4.5 & 3.5 & 4.5 \\
\hline & 2010 & 21 & 4.6 & 5.0 & 5.0 & 4.0 & 5.0 \\
\hline & 2020 & 29 & 5.7 & 5.0 & 5.0 & 5.0 & 6.0 \\
\hline \multirow[t]{4}{*}{ Lithium polymer } & 2000 & 35 & 4.8 & 5.0 & 5.0 & 4.0 & 5.0 \\
\hline & 2005 & 35 & 6.0 & 7.5 & 5.5 & 4.5 & 7.5 \\
\hline & 2010 & 35 & 7.2 & 10.0 & 6.0 & 5.0 & 10.0 \\
\hline & 2020 & 35 & 8.2 & 10.0 & 7.4 & 6.0 & 10.0 \\
\hline \multirow[t]{4}{*}{ Nickel cadmium } & 2000 & 38 & 5.7 & 5.0 & 5.0 & 5.0 & 5.9 \\
\hline & 2005 & 37 & 6.2 & 5.0 & 5.5 & 5.0 & 6.3 \\
\hline & 2010 & 37 & 6.6 & 5.0 & 6.0 & 5.0 & 6.6 \\
\hline & 2020 & 37 & 7.8 & 10.0 & 7.0 & 6.0 & 10.0 \\
\hline \multirow[t]{4}{*}{ Nickel iron } & 2000 & 21 & 8.3 & 10.0 & 9.0 & 6.0 & 10.0 \\
\hline & 2005 & 21 & 8.7 & 9.5 & 9.3 & 6.5 & 10.3 \\
\hline & 2010 & 21 & 9.1 & 9.0 & 9.5 & 7.0 & 10.5 \\
\hline & 2020 & 21 & 9.8 & 10.0 & 10.0 & 8.0 & 10.9 \\
\hline \multirow[t]{4}{*}{ Nickel metal hydride } & 2000 & 37 & 4.6 & 5.0 & 4.7 & 3.0 & 5.0 \\
\hline & 2005 & 37 & 5.3 & 5.5 & 4.9 & 3.9 & 5.5 \\
\hline & 2010 & 37 & 6.0 & 6.0 & 5.0 & 4.7 & 6.0 \\
\hline & 2020 & 36 & 6.7 & 5.0 & 6.0 & 5.0 & 7.0 \\
\hline \multirow[t]{4}{*}{ Nickel zinc } & 2000 & 20 & 3.1 & 3.0 & 3.0 & 3.0 & 3.0 \\
\hline & 2005 & 20 & 3.6 & 3.0 & 3.5 & 3.0 & 3.8 \\
\hline & 2010 & 20 & 4.2 & 3.0 & 4.0 & 3.0 & 4.6 \\
\hline & 2020 & 20 & 4.8 & 5.0 & 4.5 & 4.0 & 5.0 \\
\hline \multirow[t]{4}{*}{ Sodium sulfur } & 2000 & 39 & 4.2 & 5.0 & 4.0 & 4.0 & 5.0 \\
\hline & 2005 & 38 & 4.8 & 5.0 & 4.5 & 4.3 & 5.0 \\
\hline & 2010 & 38 & 5.4 & 5.0 & 5.0 & 4.6 & 5.0 \\
\hline & 2020 & 38 & 5.3 & 5.0 & 5.0 & 5.0 & 5.5 \\
\hline \multirow[t]{4}{*}{ Zinc air } & 2000 & 24 & 3.8 & 3.0 & 3.0 & 3.0 & 4.0 \\
\hline & 2005 & 24 & 4.3 & 3.5 & 3.5 & 3.5 & 4.2 \\
\hline & 2010 & 24 & 4.9 & 4.0 & 4.0 & 4.0 & 4.4 \\
\hline & 2020 & 24 & 5.3 & 5.0 & 4.5 & 4.0 & 5.0 \\
\hline \multirow[t]{4}{*}{ Zinc bromide } & 2000 & 20 & 3.2 & 3.0 & 3.0 & 3.0 & 3.4 \\
\hline & 2005 & 19 & 3.7 & 3.5 & 3.5 & 3.5 & 4.2 \\
\hline & 2010 & 19 & 4.2 & 4.0 & 4.0 & 4.0 & 4.9 \\
\hline & 2020 & 20 & 5.0 & 5.0 & 5.0 & 4.8 & 5.1 \\
\hline
\end{tabular}


TABLE 7 Life (in Charge/Discharge Cycles) for 10 Battery

Technologies

Battery Technology Year Obs Mean Mode Median Quartile-1 Quartile-3

\begin{tabular}{|c|c|c|c|c|c|c|c|}
\hline \multirow[t]{4}{*}{ Lead acid } & 2000 & 55 & 611 & 600 & 600 & 500 & 700 \\
\hline & 2005 & 53 & 675 & 650 & 650 & 525 & 750 \\
\hline & 2010 & 53 & 740 & 700 & 700 & 550 & 800 \\
\hline & 2020 & 53 & 872 & 800 & 800 & 600 & 1,000 \\
\hline \multirow[t]{4}{*}{ Lithium iron disulfide } & 2000 & 21 & 512 & 500 & 500 & 400 & 600 \\
\hline & 2005 & 21 & 605 & 650 & 613 & 500 & 700 \\
\hline & 2010 & 22 & 698 & 800 & 725 & 600 & 800 \\
\hline & 2020 & 31 & 884 & 1,000 & 1,000 & 800 & 1,000 \\
\hline \multirow[t]{4}{*}{ Lithium polymer } & 2000 & 36 & 577 & 500 & 500 & 500 & 600 \\
\hline & 2005 & 36 & 726 & 600 & 625 & 600 & 750 \\
\hline & 2010 & 37 & 876 & 700 & 750 & 700 & 900 \\
\hline & 2020 & 37 & 1,185 & 1,000 & 1,000 & 900 & 1,050 \\
\hline \multirow[t]{4}{*}{ Nickel cadmium } & 2000 & 42 & 1,255 & 1,000 & 1,000 & 1,000 & 1,300 \\
\hline & 2005 & 41 & 1,341 & 1,000 & 1,150 & 1,000 & 1,400 \\
\hline & 2010 & 41 & 1,428 & 1,000 & 1,300 & 1,000 & 1,500 \\
\hline & 2020 & 41 & 1,546 & 1,000 & 1,500 & 1,000 & 1,800 \\
\hline \multirow[t]{4}{*}{ Nickel iron } & 2000 & 21 & 1,055 & 1,000 & 1,000 & 1,000 & 1,127 \\
\hline & 2005 & 21 & 1,174 & 1,050 & 1,100 & 1,025 & 1,305 \\
\hline & 2010 & 21 & 1,294 & 1,100 & 1,200 & 1,050 & 1,482 \\
\hline & 2020 & 21 & 1,545 & 1,200 & 1,350 & 1,200 & 1,848 \\
\hline \multirow[t]{4}{*}{ Nickel metal hydride } & 2000 & 41 & 969 & 1,000 & 1,000 & 500 & 1,000 \\
\hline & 2005 & 41 & 1,073 & 750 & 1,050 & 550 & 1,166 \\
\hline & 2010 & 41 & 1,177 & 500 & 1,100 & 600 & 1,331 \\
\hline & 2020 & 40 & 1,312 & 500 & 1,250 & 750 & 1,506 \\
\hline \multirow[t]{4}{*}{ Nickel zinc } & 2000 & 20 & 427 & 400 & 400 & 375 & 463 \\
\hline & 2005 & 20 & 498 & 450 & 463 & 431 & 533 \\
\hline & 2010 & 20 & 570 & 500 & 525 & 488 & 603 \\
\hline & 2020 & 20 & 716 & 600 & 650 & 575 & 825 \\
\hline \multirow[t]{4}{*}{ Sodium sulfur } & 2000 & 40 & 683 & 500 & 700 & 500 & 800 \\
\hline & 2005 & 39 & 756 & 750 & 775 & 550 & 900 \\
\hline & 2010 & 39 & 829 & 1,000 & 850 & 600 & 1,000 \\
\hline & 2020 & 38 & 910 & 1,000 & 1,000 & 625 & 1,075 \\
\hline \multirow[t]{4}{*}{ Zinc air } & 2000 & 26 & 428 & 350 & 350 & 213 & 395 \\
\hline & 2005 & 25 & 498 & 375 & 400 & 306 & 447 \\
\hline & 2010 & 25 & 568 & 400 & 450 & 400 & 500 \\
\hline & 2020 & 25 & 735 & 500 & 600 & 500 & 700 \\
\hline \multirow[t]{4}{*}{ Zinc bromide } & 2000 & 20 & 560 & 600 & 600 & 500 & 600 \\
\hline & 2005 & 20 & 632 & 650 & 650 & 550 & 675 \\
\hline & 2010 & 20 & 704 & 700 & 700 & 600 & 750 \\
\hline & 2020 & 20 & 840 & 800 & 800 & 700 & 900 \\
\hline
\end{tabular}


TABLE 8 Initial Cost (in nominal $\$ / \mathbf{k W h}$ ) for 10 Battery

Technologies

Battery Technology Year Obs Mean Mode Median Quartile-1 Quartile-3

\begin{tabular}{|c|c|c|c|c|c|c|c|}
\hline \multirow[t]{4}{*}{ Lead acid } & 2000 & 54 & 185 & 200 & 190 & 150 & 200 \\
\hline & 2005 & 54 & 182 & 190 & 185 & 150 & 200 \\
\hline & 2010 & 54 & 179 & 180 & 180 & 150 & 200 \\
\hline & 2020 & 53 & 184 & 200 & 180 & 150 & 200 \\
\hline \multirow[t]{4}{*}{ Lithium iron disulfide } & 2000 & 21 & 853 & 1,000 & 850 & 800 & 1,000 \\
\hline & 2005 & 21 & 758 & 900 & 775 & 663 & 900 \\
\hline & 2010 & 22 & 664 & 800 & 700 & 525 & 800 \\
\hline & 2020 & 31 & 622 & 800 & 600 & 500 & 800 \\
\hline \multirow[t]{4}{*}{ Lithium polymer } & 2000 & 36 & 592 & 200 & 700 & 200 & 800 \\
\hline & 2005 & 36 & 499 & 175 & 550 & 175 & 650 \\
\hline & 2010 & 37 & 406 & 150 & 400 & 150 & 500 \\
\hline & 2020 & 37 & 296 & 125 & 250 & 125 & 500 \\
\hline \multirow[t]{4}{*}{ Nickel cadmium } & 2000 & 43 & 575 & 500 & 600 & 500 & 600 \\
\hline & 2005 & 43 & 546 & 450 & 550 & 450 & 600 \\
\hline & 2010 & 43 & 517 & 400 & 500 & 400 & 600 \\
\hline & 2020 & 42 & 492 & 400 & 450 & 400 & 588 \\
\hline \multirow[t]{4}{*}{ Nickel iron } & 2000 & 21 & 529 & 500 & 500 & 500 & 550 \\
\hline & 2005 & 21 & 505 & 500 & 500 & 450 & 525 \\
\hline & 2010 & 21 & 482 & 500 & 500 & 400 & 500 \\
\hline & 2020 & 21 & 448 & 500 & 464 & 400 & 500 \\
\hline \multirow[t]{4}{*}{ Nickel metal hydride } & 2000 & 43 & 569 & 600 & 583 & 350 & 600 \\
\hline & 2005 & 43 & 498 & 400 & 492 & 275 & 550 \\
\hline & 2010 & 43 & 426 & 200 & 400 & 200 & 500 \\
\hline & 2020 & 42 & 382 & 180 & 300 & 185 & 475 \\
\hline \multirow[t]{4}{*}{ Nickel zinc } & 2000 & 20 & 654 & 700 & 700 & 500 & 707 \\
\hline & 2005 & 20 & 621 & 550 & 663 & 450 & 694 \\
\hline & 2010 & 20 & 587 & 400 & 625 & 400 & 681 \\
\hline & 2020 & 20 & 548 & 300 & 600 & 300 & 650 \\
\hline \multirow[t]{4}{*}{ Sodium sulfur } & 2000 & 41 & 392 & 400 & 400 & 320 & 450 \\
\hline & 2005 & 41 & 366 & 275 & 380 & 260 & 425 \\
\hline & 2010 & 41 & 339 & 150 & 360 & 200 & 400 \\
\hline & 2020 & 40 & 318 & 150 & 333 & 188 & 400 \\
\hline \multirow[t]{4}{*}{ Zinc air } & 2000 & 25 & 483 & 500 & 500 & 300 & 545 \\
\hline & 2005 & 25 & 435 & 450 & 450 & 300 & 498 \\
\hline & 2010 & 25 & 387 & 400 & 400 & 300 & 450 \\
\hline & 2020 & 25 & 339 & 300 & 350 & 300 & 400 \\
\hline \multirow[t]{4}{*}{ Zinc bromide } & 2000 & 20 & 667 & 800 & 745 & 600 & 763 \\
\hline & 2005 & 20 & 621 & 750 & 676 & 550 & 731 \\
\hline & 2010 & 20 & 576 & 700 & 606 & 500 & 700 \\
\hline & 2020 & 20 & 523 & 600 & 567 & 400 & 600 \\
\hline
\end{tabular}


Fuel and maintenance costs in Table 3 are computed by multiplying the respondent-specified ratios by 5.8 cents. This base value of 5.8 cents $/ \mathrm{km}$ represents the average fuel, lubrication, tire, and maintenance cost for a conventional car in 1993 [AAMA, 1996]. Battery replacement costs are excluded for the EV and the three HEVs. The conventional ICE is expected to have a fuel and maintenance cost advantage in 2000 . The EV will have a slight advantage over the conventional vehicle by 2010 and a $14 \%$ ( 1.5 cents) advantage by 2020 . HEV technologies are projected to have higher fuel and maintenance costs than the conventional vehicle through the year 2020. Only the ICE-powered HEV will come within $5 \%$ of the conventional vehicle cost by 2020 .

\section{BATTERY PERFORMANCE AND COST}

\section{BATTERY TECHNOLOGIES - Respondents}

were asked to provide achievable values for key characteristics of 10 promising battery technologies. They provided estimates for five characteristics: specific energy $(\mathrm{Wh} / \mathrm{kg})$, specific power $(\mathrm{W} / \mathrm{kg})$, shelf life (year), life in charge/discharge cycles, and initial cost ( $\$ / \mathrm{kWh})$. Tables 4-8 show a summary of the responses on battery characteristics. Many respondents chose not to respond to the battery technology questions, citing their lack of expertise in the area. Compared with the participation rate of nearly $95 \%$ for basic vehicle characteristics, the battery technology participation rate was low. Lead acid technology had the highest participation rate, nearly $60 \%$. Four technologies, lithium polymer, nickel cadmium, nickel metal hydride, and sodium sulfur, had participation rates in the range of $42-48 \%$.

Lithium polymer, zinc air, lithium iron disulfide, and sodium sulfur batteries have high specific energy, while the lead acid battery has the lowest. Lithium iron disulfide, nickel zinc, lead acid, and nickel cadmium batteries have high specific power, while zinc air and zinc bromide batteries have low specific power. Nickel iron, lithium polymer, nickel cadmium, and nickel metal hydride batteries have high shelf lives, while nickel zinc and lead acid batteries have low shelf lives. The sodium sulfur battery showed a small ( 0.1 year) decline in mean shelf life between 2010 and 2020. Nickel cadmium, nickel iron, and nickel metal hydride batteries are projected as being able to go through a high number of charge and discharge cycles. The lead acid battery has the lowest initial cost, while the lithium iron disulfide battery has the highest. The battery characteristics indicate that no one battery technology is superior in all respects.

\section{INITIAL BATTERY PACK CHARACTERISTICS} FOR THE EV - An EV may be characterized to match either a desired power level (acceleration capability) or a desired range. Because the currently available batteries are energy-limited, range may be a good criterion for characterizing an EV in the near term. However, the
Delphi study used in our analysis is intended to project a long-term outlook, and therefore either mean power or mean range requirements may be used to characterize an EV. We first evaluated the initial battery pack characteristics on the basis of mean range requirements. Table 9 lists estimated power and battery pack mass necessary to meet the mean range requirements for the 10 battery technologies. Under this approach, a few battery technologies provide more power than required and have very high mass. Also, some battery technologies provide less than the mean power required and thus would not meet the performance (acceleration) requirements. Battery technologies that have low specific power and high specific energy show low power (inability to meet the acceleration requirements), while those with high specific power and low specific energy have very high mass (incompatibility with the overall vehicle characteristics). It was concluded that the initial battery pack characteristics for an EV should be evaluated on the basis of mean power requirements (listed in Table 1). The EV thus characterized will meet the acceleration requirements (will be road-worthy) and can be evaluated for other resultant characteristics, such as range, mass, and cost.

Table 10 lists the estimated initial battery pack characteristics for each battery technology for the four future years. Battery mass, energy, and initial cost are computed from the mean of responses to match the mean power requirements. EV range and battery life (in $\mathrm{km}$ ) are computed by using information from related studies [Marr, 1994; Wang, 1994]. We used a set of baseline EV energy demand (from the battery pack) and corresponding battery mass estimates by Marr to compute range. We applied a $3.3 \%$ rate of change in energy consumption per $10 \%$ change in battery mass. This $3.3 \%$ rate of change in energy consumption is half the rate of change used for total vehicle mass [OTA, 1991]. Lifetime distance in kilometers represents the shorter of two distances: (a) shelf life times annual travel $(17,600 \mathrm{~km})$ and (b) number of cycles times half the range.

The initial battery pack characteristics estimated in this fashion for EVs do not match well with the basic vehicle characteristics specified by respondents in Table 1. Nearly all respondents, 92 of 93 , answered the EV range question. They projected high range $(179 \mathrm{~km}$ in 2000 , increasing to $358 \mathrm{~km}$ in 2020) and nearly stable purchase price. Six of the 10 battery technologies can provide a $179-\mathrm{km}$ range in 2000 . The least expensive of these six batteries, nickel metal hydride, will cost $\$ 16,750$. Subtracting this initial battery cost from the mean EV purchase price of $\$ 34,300$ (see Table 2) leaves $\$ 17,550$ for the remaining components, including an expensive controller [Cuenca and Gaines, 1996]. Respondents viewed three battery technologies as capable of providing a range of $358 \mathrm{~km}$ or more in 2020 . The sodium sulfur battery is the least expensive of these 


\section{TABLE 9 Initial EV Battery Pack Power and Mass for the Mean Range ${ }^{\text {a }}$}

\begin{tabular}{|c|c|c|c|c|c|c|c|c|}
\hline \multirow[b]{2}{*}{ Battery Technology } & \multicolumn{4}{|c|}{ EV Power (kW) } & \multicolumn{4}{|c|}{ Battery Pack Mass (kg) } \\
\hline & 2000 & 2005 & 2010 & 2020 & 2000 & 2005 & 2010 & 2020 \\
\hline Lead acid & 153 & 223 & 306 & 485 & 989 & 1,303 & 1,610 & 2,265 \\
\hline Lithium iron disulfide & 44 & 59 & 75 & 10 & 266 & 319 & 358 & 404 \\
\hline Lithiu & 31 & 39 & 46 & 6 & 230 & 263 & 276 & 314 \\
\hline Nickel cadmium & 103 & 142 & 185 & 290 & 545 & 730 & 930 & 1,391 \\
\hline Nickel iron & 82 & 116 & 156 & 255 & 656 & 878 & 1,115 & 1,675 \\
\hline tal hydride & 64 & 84 & 104 & 156 & 387 & 483 & 568 & 765 \\
\hline Nickel zinc & 84 & 114 & 147 & 222 & 494 & 632 & 764 & 1,036 \\
\hline Sodium sulfur & 40 & 53 & 67 & 98 & 277 & 357 & 438 & 613 \\
\hline Zinc a & 21 & 27 & 34 & 51 & 225 & 274 & 312 & 417 \\
\hline Zinc bromide & 42 & 60 & 81 & 126 & 450 & 590 & 732 & 1,014 \\
\hline
\end{tabular}

a The mean range requirements are $179 \mathrm{~km}$ in $2000,225 \mathrm{~km}$ in 2005, $270 \mathrm{~km}$ in 2010 , and $358 \mathrm{~km}$ in 2020.

three at $\$ 21,120$. The mean vehicle price in 2020 is $\$ 35,700$, leaving $\$ 14,580$ for the remaining components.

EVs are projected to become lighter over time. Their mean curb weight is $1,538 \mathrm{~kg}$ in 2000 , dropping to $1,222 \mathrm{~kg}$ in 2020 (Table 1). On the other hand, the initial battery pack weight increases from $410 \mathrm{~kg}$ (nickel metal hydride) in 2000 to $620 \mathrm{~kg}$ (sodium sulfur) in 2020.

Some inconsistency between basic vehicle characteristics and battery characteristics appears to exist in the responses. However, almost all respondents provided information on the desired EV characteristics, while only those familiar with the battery technologies responded to the battery questions. The mean basic vehicle characteristics appear to have been influenced by the respondents who were familiar with the vehicle characteristics, but not with the battery technologies.

\section{EV BATTERY REPLACEMENT COSTS - On} average, a conventional vehicle lasts $12-15$ years and travels 170,000-210,000 km [Davis, 1995; Mintz, Tompkins, and Camp, 1994]. Because an EV does not idle while stopped and has fewer parts that are subjected to continuous wear and tear, we assumed an EV to be in use for $195,000-240,000 \mathrm{~km}, 15 \%$ longer. Since the initial battery pack has a shorter life than this, one or more replacement packs will be needed.

We computed battery replacement costs by using a sequential procedure. First, we computed intermediate values for battery specific energy, specific power, shelf life, cycles, and initial cost through linear interpolation. Next, we computed battery pack mass, energy, and cost for each of the 15 years an EV is in use for the mean power rating of the initial year (viz., 2000, 2005,2010 , or 2020 ). Next, we computed range and useable life in kilometers over a battery's shelf life and cycle life; then we computed battery replacement cost over both shelf and cycle lives and discounted it at $4 \%$ (real). Since battery technologies improve over time, the replacement batteries will have improved characteristics. For example, a year $2000 \mathrm{EV}$ will require one or more replacement battery pack(s) over its life time (during the period 2003-2015). The projected improvements in battery characteristics will offer the buyer of a replacement battery pack two alternatives: (1) buy a battery pack that delivers enough power to match the motor's rating or (2) buy as big a battery pack as fits in the space available. The second alternative will cost more, but it will provide greater range because of improvements in the battery's specific energy. We assumed that the motor's power rating will be the constraining factor when purchasing a replacement battery (i.e., to keep costs down, an EV owner will not buy a battery pack with more power, even though doing so would increase the vehicle range). Finally, we summed the discounted replacement costs, distributed them over the respective lives (i.e., shelf life or cycles), and selected the higher of the two costs. The procedure excludes the cost of the initial battery pack because the Delphi questionnaire asked the respondents to include it in vehicle purchase price.

Figure 1 shows battery replacement cost and initial pack range for the 10 technologies. Four points are plotted for each technology, representing values for EVs produced in $2000,2005,2010$, and 2020 . In general, the replacement cost declined over time and the range increased. One exception is the sodium sulfur battery, for which the replacement cost is not projected to drop after 2010 (though range increases, a bigger battery pack is necessary to match the higher power rating in 2020). The changes in replacement cost are remarkable because they are visible even with the increases in mean power requirements. Only two batteries, lead acid and nickel cadmium, are projected to have their replacement costs under 6 cents $/ \mathrm{km}$ in 2000 and 2005. The nickel iron battery will join them in 2010 
TABLE 10 Characteristics of Initial EV Battery Pack ${ }^{a, b}$

\begin{tabular}{|c|c|c|c|c|c|c|}
\hline Battery Technology & Year & $\begin{array}{l}\text { Mass } \\
(\mathbf{k g})\end{array}$ & $\begin{array}{l}\text { Energy } \\
\text { (Wh) }\end{array}$ & $\begin{array}{c}\text { Initial } \\
\text { Cost (\$) }\end{array}$ & $\begin{array}{l}\text { Range } \\
(\mathbf{k m})\end{array}$ & $\begin{array}{r}\text { Life } \\
\text { (km) }\end{array}$ \\
\hline \multirow[t]{4}{*}{ Lead acid } & 2000 & 430 & 17.040 & 3,150 & 110 & 32,100 \\
\hline & 2005 & 440 & 18.530 & 3,370 & 110 & 38,400 \\
\hline & 2010 & 450 & 20.030 & 3,590 & 120 & 45,200 \\
\hline & 2020 & 460 & 22.340 & 4,120 & 140 & 59,400 \\
\hline \multirow[t]{4}{*}{ Lithium iron disulfide } & 2000 & 400 & 38.700 & 33,010 & 250 & 61,500 \\
\hline & 2005 & 410 & 43,160 & 32,310 & 270 & 71,600 \\
\hline & 2010 & 410 & 47.610 & 31,610 & 300 & 81,800 \\
\hline & 2020 & 370 & 50.630 & 31,480 & 330 & 101,300 \\
\hline \multirow[t]{4}{*}{ Lithium polymer } & 2000 & 490 & 53.900 & 31,900 & 320 & 84,800 \\
\hline & 2005 & 500 & 64.100 & 31,040 & 370 & 106,300 \\
\hline & 2010 & 520 & 74.290 & 30,170 & 430 & 127,700 \\
\hline & 2020 & 510 & 88.100 & 26,040 & 520 & 145,000 \\
\hline \multirow[t]{4}{*}{ Nickel cadmium } & 2000 & 350 & 20,020 & 11,520 & 130 & 82,600 \\
\hline & 2005 & 390 & 22.970 & 12,460 & 150 & 98,200 \\
\hline & 2010 & 430 & 25,930 & 13,410 & 160 & 114,900 \\
\hline & 2020 & 480 & 29.660 & 14,600 & 180 & 138,300 \\
\hline \multirow[t]{4}{*}{ Nickel iron } & 2000 & 530 & 27,240 & 14,400 & 160 & 81,900 \\
\hline & 2005 & 570 & 30,530 & 15,350 & 170 & 99,300 \\
\hline & 2010 & 610 & 33,820 & 16,310 & 180 & 118,400 \\
\hline & 2020 & 650 & 37,510 & 16,800 & 200 & 153,800 \\
\hline \multirow[t]{4}{*}{ Nickel metal hydride } & 2000 & 410 & 29,440 & 16,750 & 190 & 82,000 \\
\hline & 2005 & 440 & 34,180 & 16,670 & 210 & 94,000 \\
\hline & 2010 & 470 & 38,930 & 16,590 & 230 & 106,100 \\
\hline & 2020 & 490 & 43,570 & 16,670 & 260 & 117,700 \\
\hline \multirow[t]{4}{*}{ Nickel zinc } & 2000 & 390 & 23,670 & 15,480 & 150 & 32,200 \\
\hline & 2005 & 420 & 27,040 & 16,670 & 170 & 42,000 \\
\hline & 2010 & 450 & 30,410 & 17,850 & 190 & 53,100 \\
\hline & 2020 & 460 & 34,050 & 18,660 & 210 & 74,300 \\
\hline \multirow[t]{4}{*}{ Sodium sulfur } & 2000 & 460 & 44,040 & 17,250 & 260 & 74,800 \\
\hline & 2005 & 510 & 50,770 & 18,380 & 290 & 84,900 \\
\hline & 2010 & 560 & 57,510 & 19,520 & 320 & 94,900 \\
\hline & 2020 & 620 & 66,340 & 21,120 & 360 & 93,100 \\
\hline \multirow[t]{4}{*}{ Zinc air } & 2000 & 730 & 85,110 & 41,120 & 420 & 66,500 \\
\hline & 2005 & 760 & 97,140 & 41,670 & 470 & 76,800 \\
\hline & 2010 & 800 & 109,170 & 42,220 & 520 & 87,200 \\
\hline & 2020 & 810 & 118,960 & 40,350 & 570 & 94,300 \\
\hline \multirow[t]{4}{*}{ Zinc bromide } & 2000 & 710 & 48,740 & 32,510 & 250 & 56,500 \\
\hline & 2005 & 740 & 53,410 & 32,970 & 260 & 65,500 \\
\hline & 2010 & 780 & 58,090 & 33,440 & 280 & 74,600 \\
\hline & 2020 & 800 & 63,410 & 33,160 & 310 & 87,800 \\
\hline
\end{tabular}

a For the mean power requirements of $66.7 \mathrm{~kW}$ in $2000,76.4 \mathrm{~kW}$ in $2005,86.1 \mathrm{~kW}$ in 2010 , and $99.2 \mathrm{~kW}$ in 2020.

b Values are rounded to the nearest ten or hundred. 
and nickel metal hydride in 2020 . The respondents have high expectations for the lithium polymer battery. Its replacement cost dropped from 12 cents $/ \mathrm{km}$ in 2000 to 6.8 cents $/ \mathrm{km}$ in 2020 , and it has nearly twice the range of the nickel metal hydride battery.

Results of the replacement cost analysis show the nickel metal hydride battery as capable of meeting the year 2000 mean-range requirement of $179 \mathrm{~km}$ at a cost of 7.5 cents $/ \mathrm{km}$. The nickel cadmium battery has a lower cost, 6 cents $/ \mathrm{km}$, but provides only a $130-\mathrm{km}$ range. The lithium polymer battery improves its replacement cost by $26 \%$ between 2000 and 2005 and $19 \%$ between 2005 and 2010 . The lithium polymer battery can also meet the mean-range requirements for 2005,2010 , and 2020 at the cost of $8.8,7.1$, and 6.8 cents $/ \mathrm{km}$, respectively.

As explained earlier, each battery pack in Table 10 and Figure 1 is characterized to meet the mean power requirements emerging from the Delphi data. The Delphi respondents specified higher vehicle power requirements in 2010 and 2020 , thereby implicitly requiring bigger battery packs if the battery technologies that have low specific power are to be used. A nickel metal hydride battery pack with $66.7 \mathrm{~kW}$ of power in 2000 will weigh $410 \mathrm{~kg}$, contributing an estimated $26 \%$ of the EV curb weight. A nickel cadmium battery pack with a shorter range $(130 \mathrm{~km})$ will weigh $350 \mathrm{~kg}$ and contribute $23 \%$ of the EV curb weight. Lithium polymer battery packs are estimated to be heavier, in the range of $500-520 \mathrm{~kg}$; the increased mass results from the lower specific power rating of the technology. If the range expectations are lowered to $260 \mathrm{~km}$ or less, the nickel metal hydride battery can meet them at costs lower than those of the lithium polymer battery. Even the nickel metal hydride battery packs will weigh more (see Table 10) because of increases in the mean power requirements.

The mean power requirements of $66.7,76.4$, 86.1 , and $99.2 \mathrm{~kW}$ and mean curb weights of 1,538 , $1,444,1,351$, and $1,222 \mathrm{~kg}$ for the four future years were obtained from the basic vehicle characteristics responses. These numbers translate to $0.043,0.053$, 0.064 , and $0.081 \mathrm{~kW} / \mathrm{kg}$, compared with the current desirable power-to-mass ratio of $0.074 \mathrm{~kW} / \mathrm{kg}$ $(0.045 \mathrm{hp} / \mathrm{lb})$ for the conventional ICE. Cars had average power-to-mass ratios of $0.053 \mathrm{~kW} / \mathrm{kg}(0.032$ $\mathrm{hp} / \mathrm{lb}$ ) in 1981 and 1982 [Heavenrich and Hellman, 1996]. Individual models with even lower power-to-mass ratios were acceptable during the past energy price shocks. For example, the 1982 four-door Chevrolet Chevette equipped with a diesel engine had a power-to-mass ratio of $0.037 \mathrm{~kW} / \mathrm{kg}$ [Automotive News, 1982]. Thus, although future EVs appear to be underpowered through 2010 , their power-to-mass ratios are not unrealistic.

Among the battery technologies that have replacement cost under 10 cents $/ \mathrm{km}$, lead acid is the least expensive with very limited range, and lithium polymer is the most expensive, but with high range (see Figure 1). For a better balance between power and range, a combination battery pack of lead acid and lithium polymer batteries is the best combination. Advances in battery monitoring technology are predicted to make such mixing and matching of batteries feasible in portable computing [McCormick, 1996]. We analyzed hypothetical combination battery packs in which both lead acid and lithium polymer batteries would power an $E V$ in the year 2020. The cost objective worked consistently, but power was low. We lowered the power requirement to $85 \mathrm{~kW}$, assuming that the better speedtorque relationship of an electric motor would not require as high a power-to-mass ratio as an ICE. The results of the analysis are shown in Figure 2. Two curves, median and optimistic, are shown. The median curve shows results with the median values of Delphi responses, while the optimistic curve shows results with optimistic values for specific power, specific energy, and cost. The median responses show power increasing and range declining with increased share of the battery pack by lead acid batteries. The optimistic group for the lithium polymer battery expected higher specific power for it than did the optimists for the lead acid battery. This led to reductions in both power and range when the lead acid battery share increased. In both cases, the cost of the battery pack was reduced when lead acid battery share increased.

\section{INITIAL BATTERY PACK CHARACTERISTICS} FOR THE HEV - As discussed earlier, most respondents appear to assume that all hybrids are of the "range extender" type. A "range extender" HEV should provide the specified peak vehicle power while running on batteries. The battery packs will have $79.6 \mathrm{~kW}$ of power in 2000, $89.4 \mathrm{~kW}$ in 2005, $99.1 \mathrm{~kW}$ in 2010, and $108.6 \mathrm{~kW}$ in 2020 . The resulting power-to-mass ratios (computed by using data from Table 1) are 0.051$0.086 \mathrm{~kW} / \mathrm{kg}$. Table 11 summarizes the estimated characteristics of the initial HEV battery packs for the 10 battery technologies. The estimates in the table are based on mean values of survey responses for such battery attributes as specific power, specific energy, and initial costs. For computing the range, we modified the baseline estimates by Marr [Marr, 1994]. We assumed that an HEV would consume the same amount of energy per kilometer as an EV and that its baseline battery pack mass would be similar to the baseline EV battery pack mass. Here, too, we used a $3.3 \%$ rate of change in energy consumption per $10 \%$ change in the battery pack mass. Lifetime distance in the table represents the distance computed either on the basis of shelf life or cycle life; the shorter of the two distances is shown. The first is computed as shelf life times the annual travel $(17,600 \mathrm{~km})$, while the second is the larger of (a) cycles divided by 365 times annual travel and (b) number of cycles times half the range. 


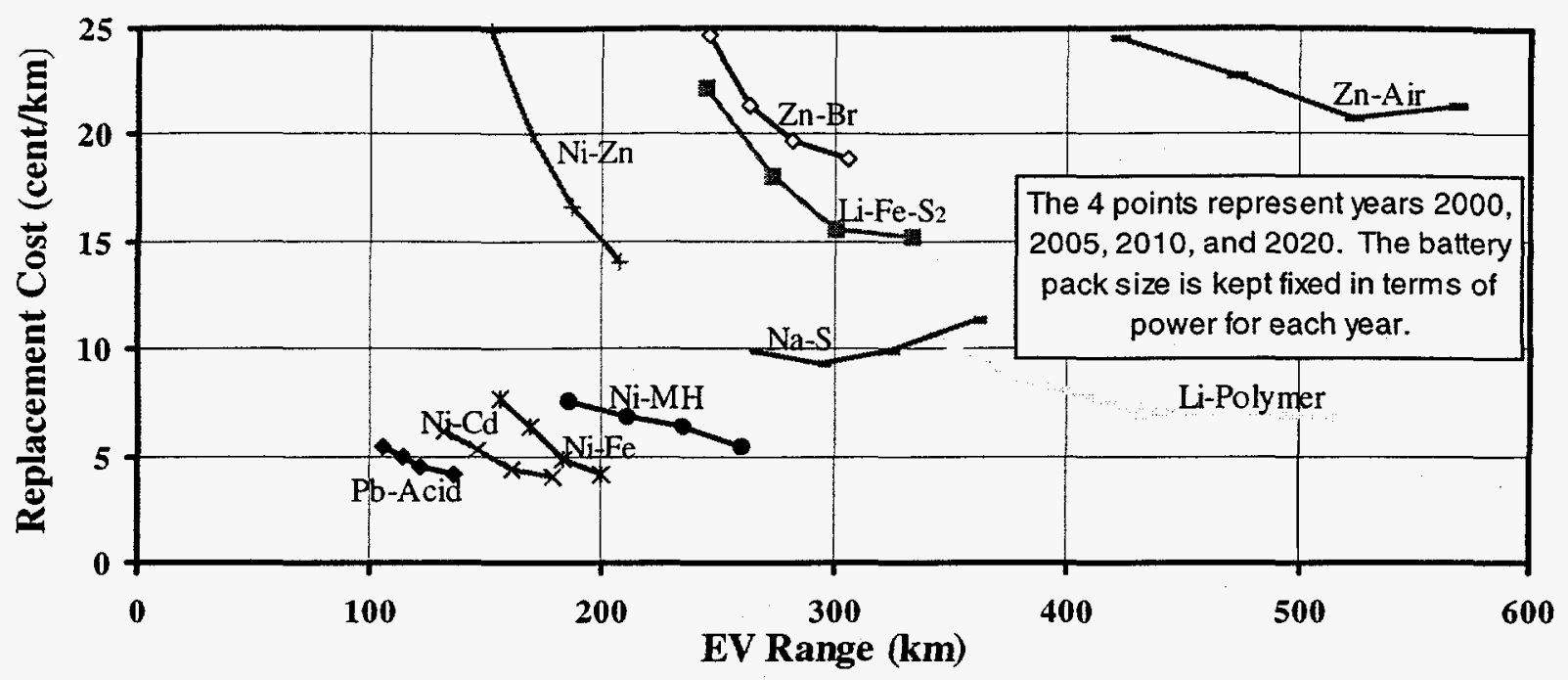

FIGURE 1. EV Battery Replacement Cost and Range Associated with the Mean Power Requirements

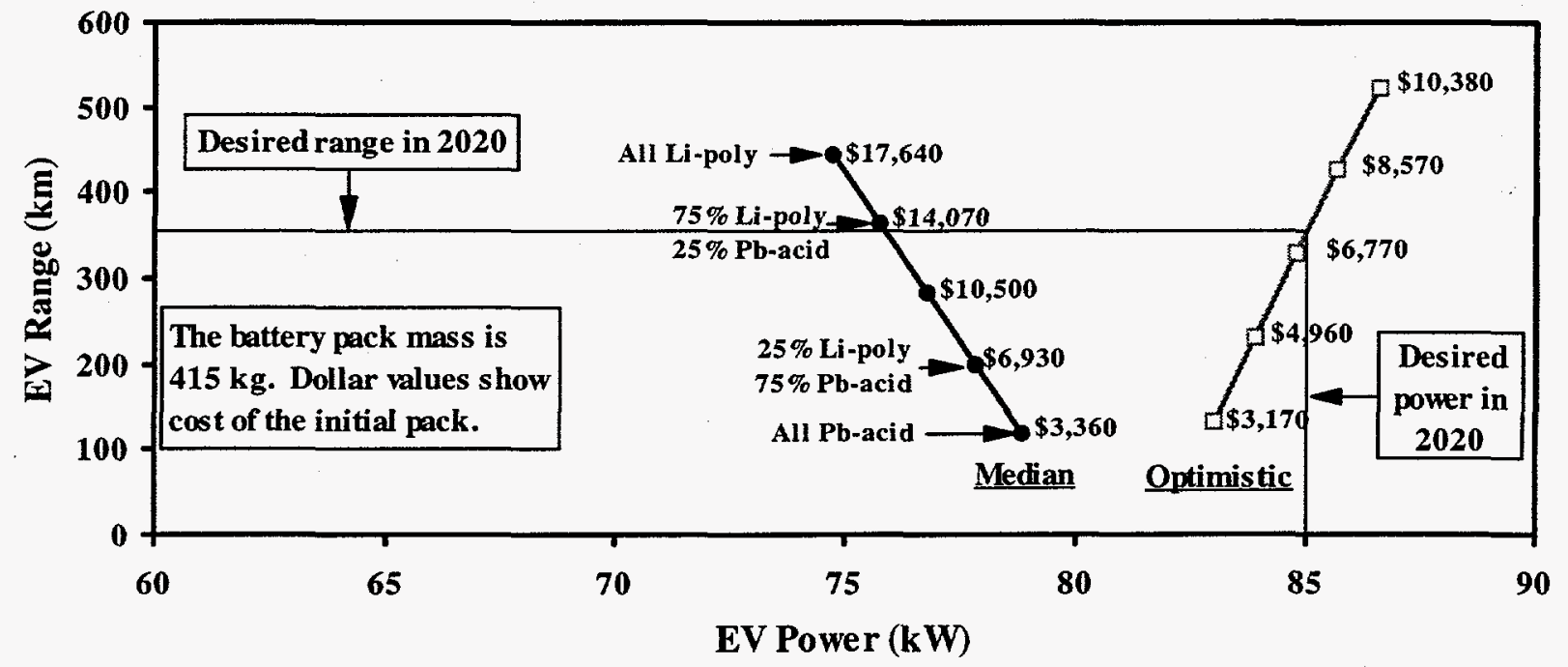

FIGURE 2. Analysis of Combined Lead-Acid and Lithium-Polymer Battery Packs for EV in 2020 
TABLE 11 Characteristics of Initial HEV Battery Pack ${ }^{a, b}$

\begin{tabular}{|c|c|c|c|c|c|c|}
\hline Battery Technology & Year & $\begin{array}{r}\text { Mass } \\
(\mathrm{kg}) \\
\end{array}$ & $\begin{array}{r}\text { Energy } \\
(W h)\end{array}$ & $\begin{array}{r}\text { Initial } \\
\text { Cost }(\$)\end{array}$ & $\begin{array}{r}\text { Range } \\
(\mathbf{k m})\end{array}$ & $\begin{array}{r}\text { Life } \\
(\mathbf{k m}) \\
\end{array}$ \\
\hline \multirow[t]{4}{*}{ Lead acid } & 2000 & 510 & 20,330 & 3,760 & 120 & 35,900 \\
\hline & 2005 & 520 & 21,690 & 3,940 & 130 & 42,400 \\
\hline & 2010 & 520 & 23,060 & 4,130 & 130 & 49,400 \\
\hline & 2020 & 510 & 24,460 & 4,510 & 140 & 62,800 \\
\hline \multirow[t]{4}{*}{ Lithium iron disulfide } & 2000 & 480 & 46,190 & 39,390 & 270 & 61,500 \\
\hline & 2005 & 480 & 50,490 & 37,890 & 300 & 71,600 \\
\hline & 2010 & 470 & 54,790 & 36,390 & 330 & 81,800 \\
\hline & 2020 & 400 & 55,430 & 34,460 & 350 & 101,300 \\
\hline \multirow[t]{4}{*}{ Lithium polymer } & 2000 & 580 & 64,320 & 38,070 & 350 & 84,800 \\
\hline & 2005 & 590 & 74,920 & 36,400 & 410 & 106,300 \\
\hline & 2010 & 590 & 85,510 & 34,730 & 470 & 127,700 \\
\hline & 2020 & 560 & 96,450 & 28,510 & 550 & 145,000 \\
\hline \multirow[t]{4}{*}{ Nickel cadmium } & 2000 & 420 & 23,890 & 13,750 & 150 & 93,200 \\
\hline & 2005 & 460 & 26,870 & 14,590 & 160 & 108,800 \\
\hline & 2010 & 500 & 29,840 & 15,430 & 180 & 116,900 \\
\hline & 2020 & 520 & 32,470 & 15,980 & 190 & 138,800 \\
\hline \multirow[t]{4}{*}{ Nickel iron } & 2000 & 640 & 32,510 & 17,190 & 170 & 90,800 \\
\hline & 2005 & 670 & 35,720 & 17,980 & 180 & 108,600 \\
\hline & 2010 & 710 & 38,930 & 18,770 & 200 & 127,900 \\
\hline & 2020 & 710 & 41,070 & 18,390 & 210 & 161,600 \\
\hline \multirow[t]{4}{*}{ Nickel metal hydride } & 2000 & 480 & 35,130 & 19,990 & 210 & 82,000 \\
\hline & 2005 & 510 & 39,970 & 19,540 & 230 & 94,000 \\
\hline & 2010 & 540 & 44,810 & 19,090 & 260 & 106,100 \\
\hline & 2020 & 530 & 47,700 & 18,250 & 280 & 117,700 \\
\hline \multirow[t]{4}{*}{ Nickel zinc } & 2000 & 470 & 28,250 & 18,480 & 170 & 36,200 \\
\hline & 2005 & 490 & 31,620 & 19,510 & 190 & 46,500 \\
\hline & 2010 & 520 & 35,000 & 20,540 & 200 & 58,000 \\
\hline & 2020 & 510 & 37,280 & 20,430 & 220 & 78,600 \\
\hline \multirow[t]{4}{*}{ Sodium sulfur } & 2000 & 550 & 52,560 & 20,580 & 300 & 74,800 \\
\hline & 2005 & 600 & 59,370 & 21,530 & 320 & 84,900 \\
\hline & 2010 & 650 & 66,190 & 22,470 & 350 & 94,900 \\
\hline & 2020 & 680 & 72,620 & 23,120 & 380 & 93,100 \\
\hline \multirow[t]{4}{*}{ Zinc air } & 2000 & 870 & 101,570 & 49,080 & 460 & 66,500 \\
\hline & 2005 & 900 & 113,610 & 48,830 & 510 & 76,800 \\
\hline & 2010 & 920 & 125,660 & 48,590 & 560 & 87,200 \\
\hline & 2020 & 890 & 130,230 & 44,170 & 590 & 94,300 \\
\hline \multirow[t]{4}{*}{ Zinc bromide } & 2000 & 840 & 58,160 & 38,790 & 270 & 56,500 \\
\hline & 2005 & 870 & 62,510 & 38,640 & 290 & 65,500 \\
\hline & 2010 & 900 & 66,870 & 38,490 & 300 & 74,600 \\
\hline & 2020 & 870 & 69,420 & 36,300 & 320 & 87,800 \\
\hline
\end{tabular}

${ }^{a}$ For the mean power requirements of $79.6 \mathrm{~kW}$ in $2000,89.4 \mathrm{~kW}$ in 2005 , $99.1 \mathrm{~kW}$ in 2010, and $108.6 \mathrm{~kW}$ in 2020.

Values are rounded to the nearest ten or hundred. 
Eighty-five out of 93 respondents (91\%) answered the HEV range question under the basic vehicle characteristics. They specified total and engine ranges for an HEV. The resulting battery ranges are 145 $\mathrm{km}$ in 2000,178 km in 2005, $212 \mathrm{~km}$ in 2010, and 244 $\mathrm{km}$ in 2020 . Under our "range extender" design assumptions, nine battery technologies are capable of providing an all-electric range of $140 \mathrm{~km}$ or longer in 2000; of these, nickel cadmium is the least expensive at $\$ 13,750$. The mean purchase price of an ICE-powered HEV is $\$ 37,080$ in 2000 (Table 2), leaving $\$ 23,330$ for the rest of the components. Six battery technologies, lithium iron disulfide, lithium polymer, nickel metal hydride, sodium sulfur, zinc air, and zinc bromide, can provide an all-electric range of over $200 \mathrm{~km}$ in 2010 and 2020. Nickel metal hydride is the least expensive of these at $\$ 19,090$ and $\$ 18,250$. An ICE-powered HEV is projected by respondents to cost $\$ 36,550$ in 2010 and $\$ 38,050$ in 2020 , which would leave $\$ 17,460$ in 2010 and $\$ 19,800$ in 2020 for the rest of the HEV.

Respondents projected the mean HEV curb weight at $1,556 \mathrm{~kg}$ in $2000,1,469 \mathrm{~kg}$ in $2005,1,382 \mathrm{~kg}$ in 2010 , and $1,265 \mathrm{~kg}$ in 2020 . The estimated mass of a nickel cadmium battery pack is projected to be $420 \mathrm{~kg}$ in 2000 , while the nickel metal hydride battery packs would weigh $540 \mathrm{~kg}$ in 2010 and $530 \mathrm{~kg}$ in 2020 . A mid-size conventional car had a curb weight of $1,368 \mathrm{~kg}$ in 1993. The Energy Information Administration projects nearly stable transportation energy prices through 2015, increasing at a rate of $0.9 \%$ per year [EIA, 1996]. Such low growth in energy prices would not require dramatic increases in vehicle fuel economy. In a related ANL study, the conventional vehicle curb weight was estimated to drop $2 \%$ by $2000,5 \%$ by 2010 , and $7 \%$ by 2020 under a baseline scenario [Stodolsky, Vyas, and Cuenca, 1995]. Thus, the projected curb weight for the conventional mid-size car would be $1,340 \mathrm{~kg}$ in 2000 , $1,300 \mathrm{~kg}$ in 2010 , and $1,270 \mathrm{~kg}$ in 2020 . The estimated weight of an HEV without its battery pack would be 1,136 $\mathrm{kg}$ in $2000,842 \mathrm{~kg}$ in 2010 , and $735 \mathrm{~kg}$ in 2020 . These low curb weights will require increased use of lightweight materials, which will increase HEV costs.

\section{HEV BATTERY REPLACEMENT COSTS -}

Earlier, we described a procedure for computing battery replacement costs for EVs. This procedure computes battery replacement intervals, assuming 17,600 kilometers per vehicle per year and a $15 \%$ longer vehicle life (in terms of life time usage) than the conventional ICE. The procedure computes replacement costs on the basis of both shelf and cycle lives and selects the higher of the two. We computed HEV battery replacement costs by means of this procedure.

The Delphi questionnaire sought experts' opinions on the future characteristics of batteries for use in both EVs and HEVs. Though it is possible to design batteries for specific end uses (such as higher specific energy for EVs and higher specific power for HEVs), all respondents provided one set of values for both EVs and HEVs. Therefore, we used the same battery

characteristics for both vehicle types.

Figure 3 shows the computed battery replacement costs for the 10 technologies. The lead acid technology has the lowest replacement costs for 2000 and 2005. The nickel cadmium battery is ranked next, with estimated replacement costs at fractions of a cent higher. The nickel metal hydride technology provides a longer battery range, but it costs more (at least one and a half cents more per kilometer than nickel cadmium in 2020). Again, the lithium polymer technology is projected to improve dramatically between 2000 and 2020.

All battery technologies except lead acid can provide a range of $140 \mathrm{~km}$ or longer for 2000; the nickel cadmium battery has the lowest replacement cost, 6.3 cents $/ \mathrm{km}$, for this period. At 6.2 cents $/ \mathrm{km}$, the nickel iron battery would be the battery of choice for 2005 to meet the $175-\mathrm{km}$ range requirement. The nickel metal hydride battery would be the battery of choice from 2010 onward, to meet the desired range of $210 \mathrm{~km}$ and higher; its estimated replacement cost is 7.3 cents $/ \mathrm{km}$ in 2010 and 5.9 cents $/ \mathrm{km}$ in 2020 . If replacement cost were the controlling factor, the lead acid battery would be preferred through 2020 , with a replacement cost ranging from 5.8 cents $/ \mathrm{km}$ in 2000 to 4.1 cents $/ \mathrm{km}$ in 2020 . The lead acid battery is projected to have a range of 118-144 $\mathrm{km}$ through 2020. A majority of urban vehicles travel 45$48 \mathrm{~km} /$ day [Wang, 1994; NPTS, 1991]; the lead acid battery would be able to supply the necessary energy for that distance.

The U.S. government and the automotive industry have developed a partnership, the Partnership for a New Generation of Vehicles (PNGV), to develop high-fuel-economy vehicles. The need for such a vehicle arises from concerns about global warming and the fear of excessive reliance of the U.S. economy on imported oil. One of the technological options under consideration by PNGV is the development of a low all-electric range and low battery-power (or battery/ultracapacitor-power) hybrid electric vehicle. Such a vehicle may not be designed for, or expected to use, electricity from the electric grid. Any all-electric operation would be limited to low-speed (and low-acceleration) local driving and cruising. Separately, we analyzed battery replacement costs for a low-battery-power hybrid, assuming its batteries to have power equal to half the mean power specified by the Delphi respondents. We applied the same methodology, and the resulting battery replacement costs are shown in Figure 4. The pattern of battery replacement costs is similar to the "range extender" HEVs described above. Since the range on battery power is not a constraining factor for these HEVs, lead acid emerges as the least-cost battery technology, with nickel cadmium as the next near-term alternative. 


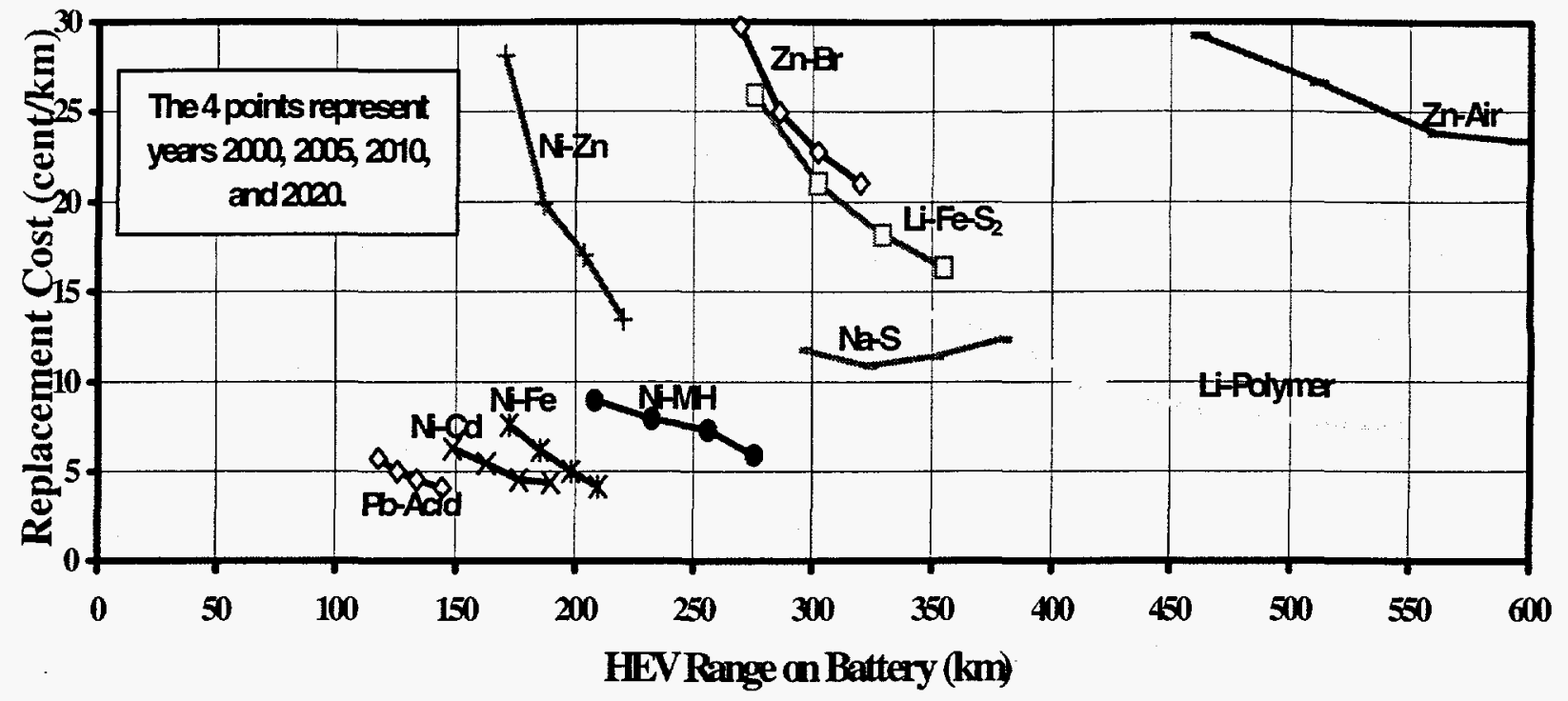

FIGURE 3. HEV Battery Replacement Cost and Range Associated with the Mean Power Requirements

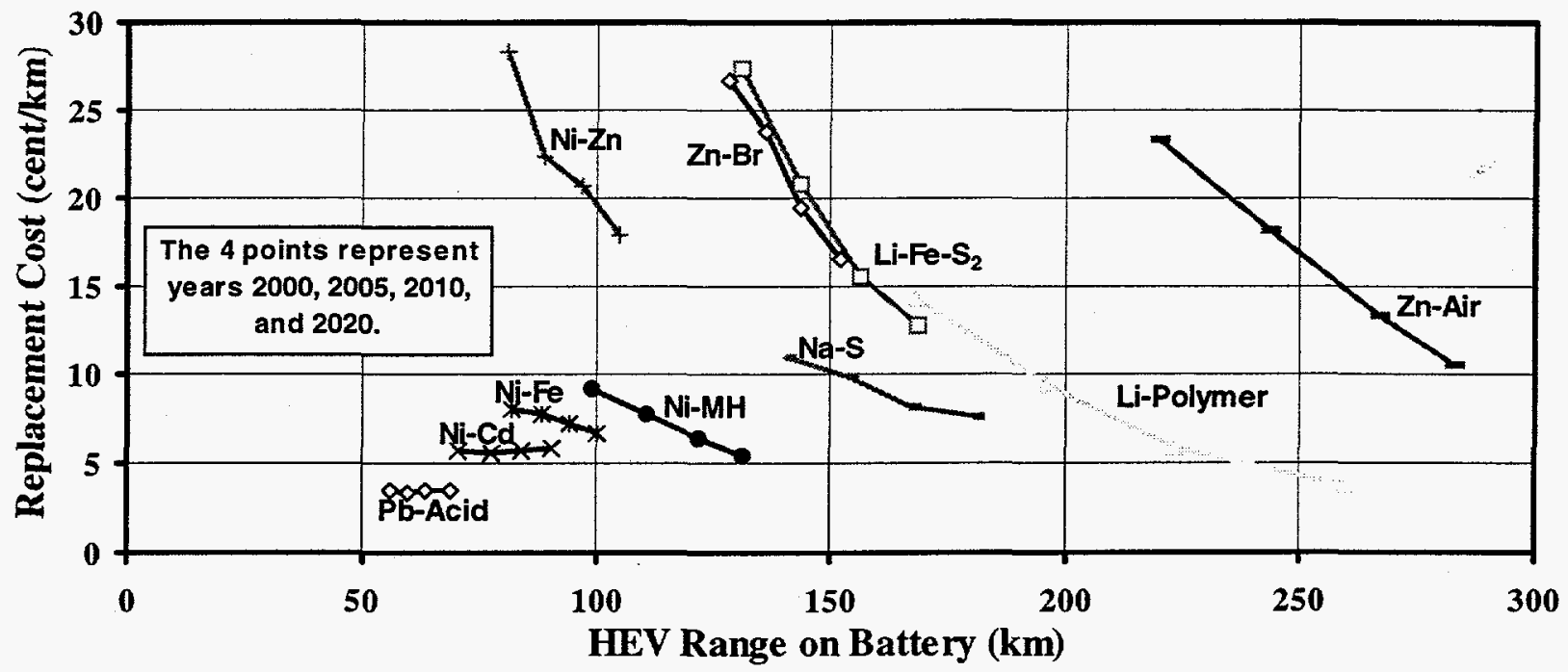

FIGURE 4. HEV Battery Replacement Cost and Range Associated with Half the Mean Power Requirements 
The lithium polymer battery was estimated by this method to be a low-cost alternative in the long term.

It appears possible, in retrospect, that the survey questionnaire's structure guided respondents to think in terms of "range extender" HEVs rather than PNGV-type HEVs. The absence of separate battery questions for EVs and HEVs, and the nature of the cycle life question, would have promoted this type of response. The cycle life question asked for cycle life to $50 \%$ state of charge (SOC). A question valid for a PNGV-type HEV might have asked for cycle life if the battery were operated from $80 \%$ to $60 \%$ SOC. Since such a question was not asked, it is not possible to estimate the pattern of battery replacement costs for a PNGV-type HEV. Note that relatively few battery responses were obtained, so any increased complexity of the questionnaire might have been unproductive in any case.

VARIABLE OPERATING COSTS, INCLUDING BATTERY REPLACEMENT - Earlier, we analyzed fuel and maintenance cost responses under electric drive vehicle characteristics. The mean fuel maintenance costs for EVs and HEVs did not include battery replacement costs. Among the three HEV technologies, the fuel-cell-powered HEV is not expected to have a large battery pack. We added the above-discussed battery replacement costs to the EV and the other two HEV technologies and compared the results with that for conventional ICE vehicles.

First, we computed total variable operating cost for an EV or HEV when the battery technology of choice meets the mean range requirements. Figure 5 shows the results of this comparison. In 2000, the EV is powered by a nickel metal hydride battery pack and the two HEVs are powered by nickel cadmium battery packs. Lithium polymer is the battery of choice for EVs from 2005 onward, while nickel iron in 2005 and nickel metal hydride from 2010 onward are the batteries of choice for HEVs. The conventional ICE has the decided advantage of low variable operating cost. The EV has a slightly higher operating cost than the ICE-powered HEV through 2005 and lower operating costs thereafter. The gasturbine-powered HEV has the highest operating cost through the analysis period. The respondents projected lower variable operating cost for the fuel-cell-powered HEV compared to the EV and other two HEVs.

We also computed total variable operating costs with reduced range expectations. We selected the nickel cadmium battery for the EV and the lead acid battery for HEVs, for all years. Although lead acid is the least expensive battery for the EV in 2000 and 2005, we did not select it because of its limited range. The results are shown graphically in Figure 6 . Total variable costs dropped, making the EV more attractive than the two HEVs. However, the conventional vehicle still has an estimated cost advantage. Only if consumers viewed emission reduction and energy independence advantages as more important than these cost differences could the new technology vehicles gain significant market shares.

\section{SUMMARY}

We have presented some results of a two-stage Delphi study concerning EVs and HEVs. Expected vehicle characteristics and the future characteristics of 10 battery technologies were summarized. We evaluated initial battery pack costs and also analyzed replacement battery costs. The results are summarized as follows:

1. A large majority, $91-98 \%$, of the 93 respondents to the second-stage Delphi questionnaire gave opinions on basic vehicle characteristics for EVs and HEVs. These characteristics include power, range, curb weight, and seating capacity.

- Respondents projected a $49 \%$ increase in mean EV power capability and a $36 \%$ increase in mean HEV power capability between 2000 and 2020.

- They projected a $100 \%$ increase in mean EV range between 2000 and 2020 . For the HEV, they projected a $49 \%$ increase in total range and a $31 \%$ increase in the engine range between 2000 and 2020 . The estimated battery-only range is $39 \%$ of the total in 2000 and $47 \%$ of the total in 2020 . The high battery ranges imply to us that the candidate HEVs are seen by respondents as "range extenders."

- They projected a $21 \%$ reduction in the mean EV curb weight and a $19 \%$ reduction in the mean HEV curb weight between 2000 and 2020 .

- They projected the EV to have fewer than five seats until 2020 and the HEV to have fewer than five seats until 2010.

2. A slightly lower number of respondents, $75-93 \%$, gave opinions on vehicle purchase price and variable operating cost, excluding batteries.

- They projected a $102 \%$ increase in the conventional vehicle price by 2020 compared to that for 1993. They projected both the EV and the HEV to have higher initial prices than the conventional vehicle through 2020.

- They projected the conventional vehicle to have an advantage in terms of variable operating cost in 2000 , even when the battery replacement cost was excluded. An EV would have a variable cost advantage beginning in 2010. All three HEV technologies would have higher variable costs compared with the conventional vehicle's through 2020. 


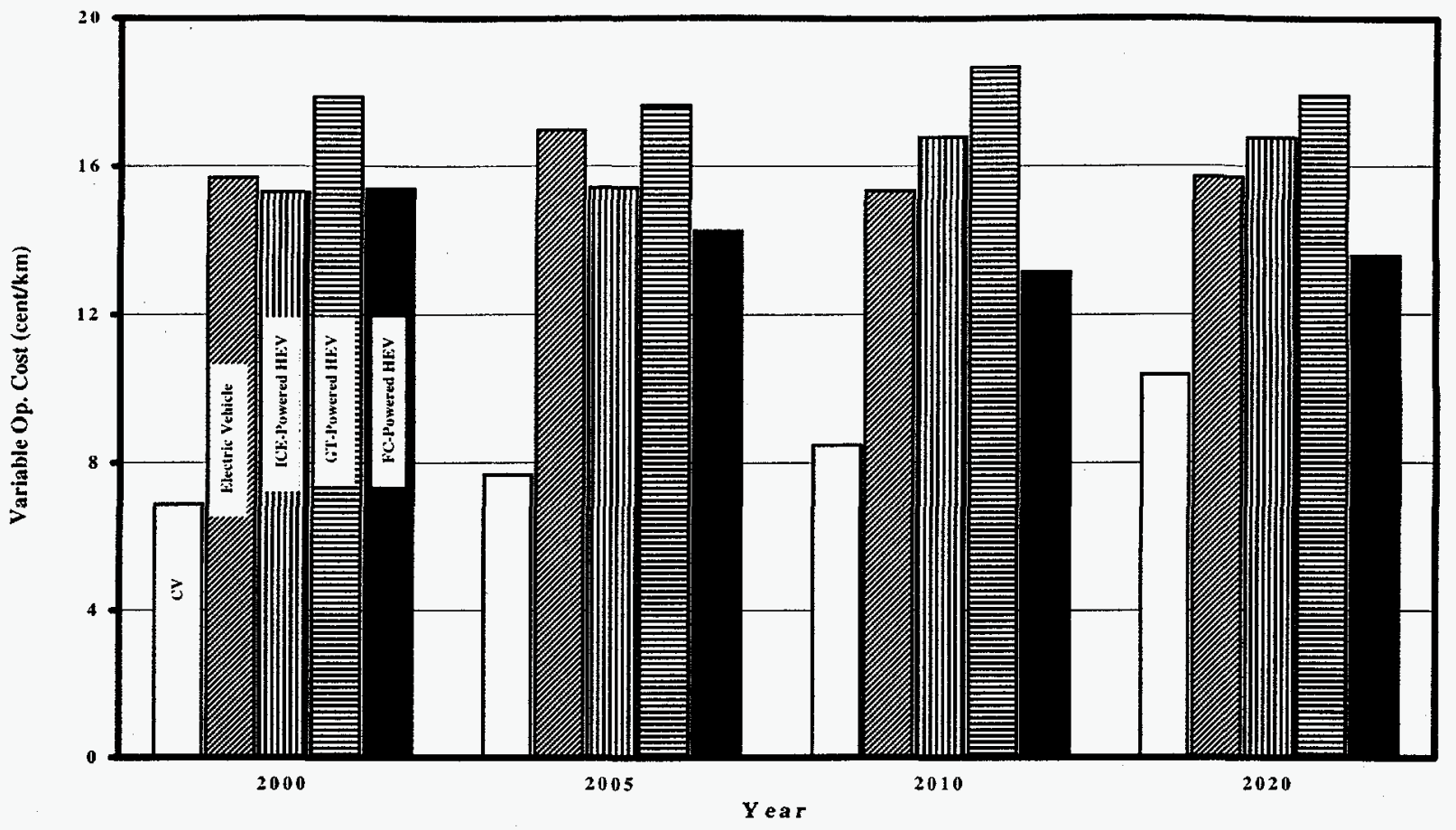

FIGURE 5. Variable Operating Costs, Including Battery Replacement Costs and Matching the Mean Range Requirements

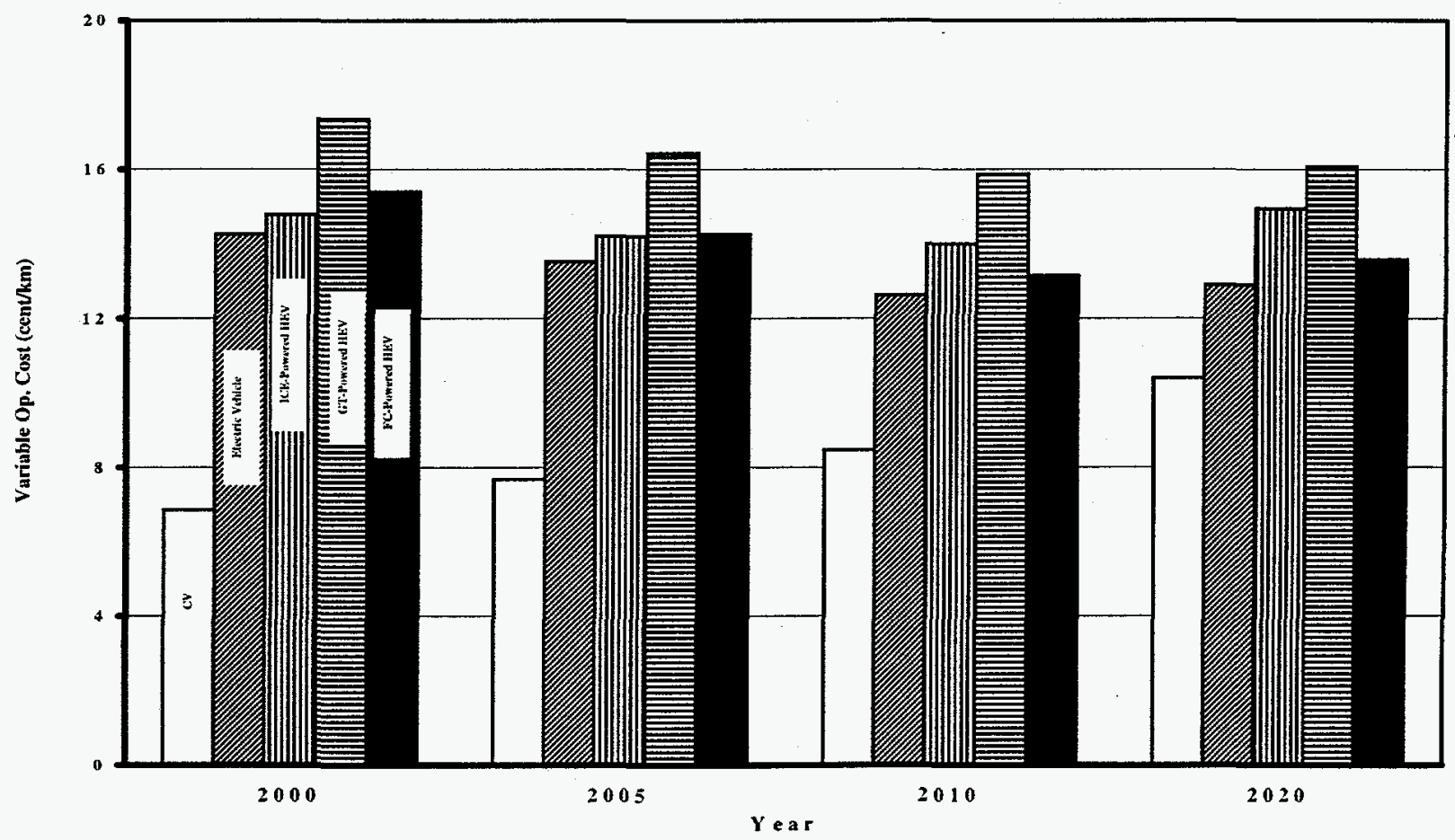

FIGURE 6 Variable Operating Costs with Less Expensive Battery, Ignoring the Mean Range Requirements 
3. A smaller number of respondents, $22-59 \%$, gave opinions on 10 battery technologies. They provided input on such future battery characteristics as specific energy, specific power, shelf life, number of charge/discharge cycles, and initial cost.

- They projected that almost all battery technologies would improve over time. The rate of improvement varies among technologies. No one battery technology is superior in all respects.

- The high mean-range requirements for EVs and HEVs will necessitate expensive battery packs, given the battery characteristics projected by respondents. The price of the initial battery pack will therefore make purchase price predictions very difficult to achieve. The mass of the initial battery is also expected to be high, making the mean curb weight predictions difficult to achieve.

4. We developed a procedure to compute battery replacement costs and computed these costs for both the EV and the HEV.

- Almost all battery technologies were projected to reduce their cost over time. The lithium polymer battery showed the highest rate of improvement.

- If the mean-range requirements are to be met, the battery replacement cost will be 6.8 cents $/ \mathrm{km}$ or higher for EVs and 5.9 cents $/ \mathrm{km}$ or higher for HEVs.

- If the EV range requirements are moderated as $130 \mathrm{~km}$ in 2000, $160 \mathrm{~km}$ in 2010, and $180 \mathrm{~km}$ in 2020 , then relatively low battery replacement costs of 6 cents $/ \mathrm{km}$ or lower are projected.

- HEV battery pack replacement costs can be reduced substantially with the use of lead acid batteries. The battery-only range will be limited to $115-145 \mathrm{~km}$, but the battery replacement cost will be reduced to 4.1-5.8 cents $/ \mathrm{km}$.

- When battery replacement costs are added to variable operating costs, the respondents' battery characteristics predictions imply that the conventional vehicle would have a substantial operating cost advantage. Both EVs and HEVs reduce their total variable costs when the mean battery range requirement predictions are ignored and the least expensive battery is selected.

\section{ACKNOWLEDGMENTS}

The authors thank Dr. Philip Patterson of the Office of Transportation Technologies, U.S. Department of Energy, for his continuing support and valuable guidance. The authors also thank Floyd Bennett for editing this paper. This work was sponsored by the
U.S. Department of Energy, Assistant Secretary for Energy Efficiency and Renewable Energy, under contract W-31-109-Eng-38.

\section{REFERENCES}

AAMA (1996), Motor Vehicle Facts \& Figures '95, American Automobile Manufacturers Association, Detroit, Mich.

ANL (1996), Electric/Hybrid Vehicle Study: Second Stage Results, Center for Transportation Research, Argonne National Laboratory, Argonne, III.

Automotive News (1982), 1982 Market Data Book Issue, Detroit, Mich.

Cuenca, R.M., and L.L. Gaines (1996), Estimates of Electric Vehicle Production Cost, Argonne National Laboratory, unpublished information.

Davis, S.C. (1995), Transportation Energy Data Book: Edition 15, Oak Ridge National Laboratory Report ORNL-6856, Oak Ridge, Tenn.

EIA (1996), Annual Energy Outlook 1996, Energy Information Administration, U.S. Department of Energy Report DOE/EIA-0383(96), Washington, D.C.

Heavenrich, R.M., and K.H. Hellman (1996), Light-Duty Automotive Technology and Fuel Economy Trends through 1996, U.S. Environmental Protection Agency Report EPAJAATDSG/96-01, Ann Arbor, Mich.

Marr, W.W. (1994), Estimates of Energy Consumption of Electric and Conventional Vehicles, Argonne National Laboratory, unpublished information.

McCormick, J. (1996), The Battle between Lithium-lon and Zinc-Air Batteries Has Begun, Government Computer News / State \& Local, Silver Springs, Md.

Mintz, M.M., M.M. Tompkins, and J. Camp (1994), The IMPACTT Model: Structure and Technical Description, Argonne National Laboratory Report ANL/ESD/TM-93, Argonne, III.

Ng, H.K., J.L. Anderson, and D.J. Santini, (1995) The Prospects for Electric/Hybrid Vehicles, 2000-2020: First-Stage Results of a Two-Stage Delphi Study, in Electric and Hybrid Vehicles - Implementation of Technology, SAE International Report SP-1105, pp. 123-135, Warrendale, Penn.

Ng, H.K., J.L. Anderson, D.J. Santini, and A.D. Vyas (1996), The Prospects for Electric and Hybrid Electric Vehicles: Second-Stage Results of a Two-Stage Delphi Study, in Technical Solutions to Alternative 
Yransportation Problems, SAE International Report SP-1189, pp. 111-130, Warrendale, Penn.

\section{NPTS (1991), 1990 Nationwide Personal}

Transportation Survey: User's Guide for the Public Use Tapes, Federal Highway Administration,

U.S. Department of Transportation, Washington, D.C.

OTA (1991), Improving Automobile Fuel Economy: New Standards, New Approaches, Office of Technology Assessment, U.S. Congress Report OTA-E-504, Washington, D.C.
Stodolsky, F., A. Vyas, and R. Cuenca (1995), Lightweight Materials in the Light-Duty Passenger Vehicle Market: Their Market Penetration Potential and Impacts, Proceedings of the Second World Car Conference, University of California at Riverside, Riverside, Calif.

Wang, M.Q. (1994), Vehicle Travel Patterns in the Four Studied Areas and Driving Cycle Selection for Simulating EV Energy Consumption and CV Energy Consumption and Emissions, Argonne National Laboratory, unpublished information. 